SISSA 02/05/EP

hep-th/0501011

\title{
Time-localized projectors in String Field Theory with E-field
}

\author{
C.Maccaferri ${ }^{1}$, R.J.Scherer Santos ${ }^{2}$, D.D.Tolla ${ }^{3}$ \\ International School for Advanced Studies (SISSA/ISAS) \\ Via Beirut 2-4, 34014 Trieste, Italy, and INFN, Sezione di Trieste
}

\begin{abstract}
We extend the analysis of hep-th/0409063 to the case of a constant electric field turned on the worldvolume and on a transverse direction of a D-brane. We show that time localization is still obtained by inverting the discrete eigenvalues of the lump solution. The lifetime of the unstable soliton is shown to depend on two free parameters: the $b$-parameter and the value of the electric field. As a by-product, we construct the normalized diagonal basis of the star algebra in $B_{\mu \nu}$-field background.
\end{abstract}

\footnotetext{
${ }^{1}$ maccafer@sissa.it

${ }^{2}$ scherer@sissa.it

3 tolla@sissa.it
} 


\section{Introduction}

The decay of non-BPS D-branes through open string Tachyon Condensation is an important phenomenon as far as time dependence in String Theory is concerned. It is clear by now that the study of Tachyon Condensation can be naturally implemented in the framework of Open (Super) String Field Theory, 1]. The theory can be formulated on an unstable D-brane, and evidence for a stable closed string vacuum (the tachyon condensate) has been given in great abundance (see [2, 3, 4, 5] for review and references therein). However, at the moment, no analytical explicit solution has been given to the classical equation of motion of OSFT, with the appropriate characteristics of the tachyon vacuum. Even more subtle is the problem of finding time dependent solutions interpolating from the unstable $\mathrm{D}$-brane to the pure closed string background arising after tachyon condensation. Some attempts towards obtaining these solutions in Cubic Open String Field Theory were made in [6]. In [7] it was shown that the time coordinate given by the center of mass in the time direction fails to be a causal choice of time for which a consistent initial values problem can be defined, in this regard it was proposed that a causal time coordinate is the midpoint component of the light-cone time. In [8] it was given some numerical evidence in (modified) level truncation that the midpoint light-cone time dependent solutions avoid exploding oscillations and converge to some finite value of the zero momentum tachyon. Despite this progress, it is still a challenge to find an exact analytic method to attack classical OSFT.

In this regard, Vacuum String Field Theory, 9, although formulated in a singular way with respect to a regular worldsheet geometry (it is supposedly obtained from Witten's OSFT by a singular field redefinition which contracts the open string to its midpoint, hence shrinking worldsheet boundaries to zero size holes), [10], is remarkably successful in describing analytically classical solutions (open string vacua), which correspond to idempotents of the matter star algebra, the ghost part of the solution being universal for all kinds of open string backgrounds, because of the universal nature of the kinetic operator. VSFT is conjectured to represent OSFT around the to-be-found tachyon vacuum and has the great advantage of having a kinetic operator which is simply a $c$-midpoint insertion. Of course one has to pay the cost of a such drastic simplification in terms of many ambiguous quantities that arise while computing observables, these ambiguities being related to midpoint singularities, which need some regularization [11, 10, 12, 13, 14. Nevertheless, it is still very attractive to consider star algebra projectors as describing, at least in the leading order of some consistent regularization scheme, static and time dependent open string vacua such as D-branes and their classical decay.

Driven by these considerations, the authors of [16] have shown that it is possible to get time localized solutions of VSFT by taking an euclidean lump solution on a transverse direction (the euclidean time) and simply inverting the discrete eigenvalues of the lump Neumann matrix. This solution, preserving the same euclidean action as the conventional lump ${ }^{4}$, has the remarkable feature of being localized in the time coordinate identified by the twist even discrete eigenvector of the Neumann matrices and, what is more important, of being localized in the center of mass time coordinate for every value of the free parameter $b$. Moreover, it was found that time dependence disappears when $b \rightarrow \infty$ (the solution

\footnotetext{
${ }^{4}$ We stress that in presence of time dependent backgrounds, one cannot anymore consider the value of the classical action mod volume as the space averaged energy
} 
becoming the zero momentum sliver state) and when $b \rightarrow 0$ (the solution becoming the 0 string field, i.e. the "stable" vacuum of VSFT). This leads the authors of [16] to propose that, at least in these singular limits, the $b$-parameter should be related to the $\tilde{\lambda}$ parameter of Sen's Rolling Tachyon BCFT. ${ }^{5}$

In the present work we are going to study the corresponding time dependent solution in the presence of a constant $E$-field background on a longitudinal or transverse direction. We obtain $E$-field physics by first going to a euclidean signature with imaginary $B$-field, $B=i E$, and then inverse Wick rotating, in the same way as [17] for what concerns the effective target space and BCFT analysis.

One of the main differences with respect to [16] is that when the $E$-field reaches its critical value $E_{c}=\frac{1}{2 \pi \alpha^{\prime}}$, the (center of mass) time dependence is lost, regardless of the $b$-parameter, and we get a flat non zero time profile which, along the lines of [17] should be interpreted as a static background of fundamental strings, polarized by the $E$-field. This result persists when $b \rightarrow 0$ if we double scale appropriately $E \rightarrow E_{c}$ with $b \rightarrow 0$. A direct construction of the fundamental string background in VSFT is proposed in [18.

The rest of this paper is organized as follows. In Section 2, we construct the solution representing the decay of a D25-brane with an E-field turned on along a longitudinal direction and show that this solution is obtained from the corresponding solution without $E$-field, where the flat metric along the direction of the $E$-field and the transverse euclidean time direction, is replaced with the open string metric. We show that in this case the $E-$ field manifests itself via the life time of the D-brane, which is longer than its value without $E$-field and can be infinite for the $E$-field approaching its critical value, at which (open) strings becomes effectively tensionless and closed strings decouple, 19. In Section 3, we construct a solution which represents the brane decay with an $E$-field turned on along a transverse direction and show that, although the $E$-field coupling to transverse momenta cannot be anymore disregarded, the physical situation is the same as the longitudinal case, but with localization on the transverse spatial direction, except at the extreme value $b=\infty$. In Section 4, we make a summary and discuss our results. In the first two appendices we carry out a detailed analysis of the spectroscopy of the Neumann matrices in $B(E)$-field background and explicitly diagonalize the 3 -string vertex. This analysis fills some gaps of [20], in particular we appropriately fix the normalizations of continuous and discrete eigenvectors and, for the latter, we give a different expression which, contrary to [20], reproduces the known results for $B=0$, 21]. In appendix $C$ we study the asymptotic behavior of Neumann matrices for the relevant limits $b \rightarrow \infty$ and $b \rightarrow 0$.

\section{Longitudinal $E$-field}

In this section we will analyze the case of switching the $E$-field along a tangential direction, i.e., along, say, the world volume of a D25-brane. As explained in [17, the presence of the $E$-field does not create non commutativity as the direction in which it is turned on is at zero momentum.

We use the double Wick rotation, that is we make space-time euclidean by sending $X^{0}(\sigma) \rightarrow i X^{D}(\sigma)$; then we construct an unconventional lump solution, [16], on the trans-

\footnotetext{
${ }^{5}$ Another approach in obtaining time dependent solutions in VSFT can be found in [15]
} 
verse spatial direction $X^{D}(\sigma)$ and inverse Wick rotate along it, $X^{D}(\sigma) \rightarrow-i X^{0}(\sigma)$. Let $\alpha, \beta=1, D$ be the couple of directions on which the $E$-field is turned on. Then $E$-field physics is obtained by taking an imaginary $B$-field

$$
B_{\alpha \beta}=B \epsilon_{\alpha \beta}=i E \epsilon_{\alpha \beta}, \quad E \in \Re
$$

A localized time dependent solution is easily given by straightforwardly changing the metric $\eta_{\alpha \beta}$ of the solution of [16], with the open string metric $G_{\alpha \beta}$

$$
\begin{aligned}
G_{\alpha \beta} & =\left(1-\left(2 \pi \alpha^{\prime} E\right)^{2}\right) \delta_{\alpha \beta} \\
G^{\alpha \beta} & =\frac{1}{1-\left(2 \pi \alpha^{\prime} E\right)^{2}} \delta^{\alpha \beta}
\end{aligned}
$$

Note that, contrary to the case of a real $B$-field, a critical value shows up for the imaginary analytic continuation ${ }^{6}$

$$
E_{c}=\frac{1}{2 \pi \alpha^{\prime}}
$$

From now on all indexes $(\alpha, \beta)$ are raised/lowered with the open string metric (2.2).

We have then the following commutators

$$
\left[a_{m}^{\alpha}, a_{n}^{\beta \dagger}\right]=G^{\alpha \beta} \delta_{m n}, \quad m, n \geq 1
$$

stating that the $a^{\alpha}$ 's are canonically normalized with respect the open string metric (2.2)

We recall that, in case of a background $B_{\alpha \beta}$-field, the three string vertex is deformed to be, 22] (see also [23])

$$
\left|V_{3}\right\rangle=\left|V_{3, \perp}\right\rangle \otimes\left|V_{3, \|}\right\rangle
$$

The factor $\left|V_{3, \|}\right\rangle$ concerns the directions with no $B$-field and its expression is the usual one, 34, 37, 35, 36], on the other hand $\left|V_{3, \perp}\right\rangle$ deals with the directions on which the $B$ field is turned on ${ }^{7}$.

$$
\left|V_{3, \perp}\right\rangle=\int d^{26} p_{(1)} d^{26} p_{(2)} d^{26} p_{(3)} \delta^{26}\left(p_{(1)}+p_{(2)}+p_{(3)}\right) \exp \left(-E^{\prime}\right)|0, p\rangle_{123}
$$

The operator in the exponent is given by, 22

$$
\begin{aligned}
E_{\perp}^{\prime}=\sum_{r, s=1}^{3} & \left(\frac{1}{2} \sum_{m, n \geq 1} G_{\alpha \beta} a_{m}^{(r) \alpha \dagger} V_{m n}^{r s} a_{n}^{(s) \beta \dagger}+\sum_{n \geq 1} G_{\alpha \beta} p_{(r)}^{\alpha} V_{0 n}^{r s} a_{n}^{(s) \beta \dagger}\right. \\
& \left.+\frac{1}{2} G_{\alpha \beta} p_{(r)}^{\alpha} V_{00}^{r s} p_{(s)}^{\beta}+\frac{i}{2} \sum_{r<s} p_{\alpha}^{(r)} \theta^{\alpha \beta} p_{\beta}^{(s)}\right)
\end{aligned}
$$

Note that the part giving rise to space-time non-commutativity, $\frac{i}{2} \sum_{r<s} p_{\alpha}^{(r)} \theta^{\alpha \beta} p_{\beta}^{(s)}$, does not contribute due to the zero momentum condition in the 1 spatial direction.

\footnotetext{
${ }^{6}$ In the rest of the paper we will set $\alpha^{\prime}=1$

${ }^{7}$ Note that in the case under consideration the symbols $\perp$ and $\|$ do not refer to perpendicular or transverse directions to the brane, but simply indicates directions with $E$-field turned on $(\perp)$ or not $(\|)$
} 
Let's first consider the sliver solution at zero momentum along the 1 direction The three string vertex in such a direction takes the form $\left(p^{1}=p_{1}=0\right)$

$$
\begin{aligned}
\left|V_{3}(E, p=0)\right\rangle & =\left|V_{3}(E=0, p=0)\right\rangle^{\left(\eta_{11} \rightarrow G(E)_{11}\right)} \\
& =\exp \left(\frac{1}{2} \sum_{r, s=1}^{3} G_{11} a^{(r) 1 \dagger} \cdot V^{r s} \cdot a^{(s) 1 \dagger}\right)|0\rangle
\end{aligned}
$$

This implies that the zero momentum sliver is in this case

$$
\begin{aligned}
|S(E, p=0)\rangle & =|S(E=0, p=0)\rangle^{\left(\eta_{11} \rightarrow G(E)_{11}\right)} \\
& =\mathcal{N} \exp \left(-\frac{1}{2} G_{11} a^{1 \dagger} \cdot S \cdot a^{1 \dagger}\right)|0\rangle
\end{aligned}
$$

where the normalization $\mathcal{N}$ and the matrix $S$ are given as usual, 30,

$$
\begin{aligned}
T & =C S=\frac{1}{2 X}(1+X-\sqrt{(1+3 X)(1-X)}) \\
\mathcal{N} & =\sqrt{\operatorname{det}(1-X)(1+T)}
\end{aligned}
$$

On the euclidean time direction we need the full 3 string vertex in oscillator basis. This is given by

$$
\left|V_{3, \perp}\right\rangle^{\prime}=K e^{-E^{\prime}}\left|\Omega_{b}\right\rangle
$$

with

$$
\begin{aligned}
K & =\left(\frac{\sqrt{2 \pi b^{3}}}{3\left(V_{00}+b / 2\right)^{2}}\left(1-(2 \pi E)^{2}\right)^{\frac{1}{2}}\right)^{\frac{1}{2}}, \\
E^{\prime} & =\frac{1}{2} \sum_{r, s=1}^{3} \sum_{M, N \geq 0} a_{M}^{(r) D \dagger} V_{M N}^{\prime r s} a_{N}^{(s) D \dagger} G_{D D}
\end{aligned}
$$

where $M, N$ denote the couple of indices $\{0, m\}$ and $\{0, n\}$, respectively, and $D$ is the (euclidean) time direction. The coefficients $V_{M N}^{\prime r s}$ are given in Appendix B of [30]. In order to have localization in Minkowski time, we need an explosive profile in euclidean time (unconventional lump); this is explained in detail in [16]

$$
\left|\check{\Lambda}^{\prime}\right\rangle=\mathcal{N} \exp \left(-\frac{1}{2} G_{D D} a^{\dagger D} C \check{T}^{\prime} a^{\dagger D}\right)\left|\Omega_{b}\right\rangle
$$

where

$$
\check{T}_{N M}^{\prime}=-\int_{-\infty}^{\infty} d k V_{N}^{(k)} V_{M}^{(k)} \exp \left(-\frac{\pi|k|}{2}\right)+\left(V_{N}^{(\xi)} V_{M}^{(\xi)}+V_{N}^{(\bar{\xi})} V_{M}^{(\bar{\xi})}\right) \exp |\eta|
$$

We refer to [16] for the exact definition of eigenvalues and eigenvectors of the various Neumann matrices in the game. We only stress that the Neumann matrix of the unconventional lump has inverted discrete eigenvalues with respect to the ordinary lump: this, as shown, 
in [16, is what guarantees time localization with respect to the center mass and to the time coordinates identified by the discrete eigenvectors $V_{N}^{(\bar{\xi})}, V_{N}^{\xi)}$.

We get a localized time profile by projecting on the coordinates/momenta of the discrete spectrum

$$
\begin{aligned}
& \hat{x}_{\eta}=\frac{i}{\sqrt{2}}\left(e_{\eta}-e_{\eta}^{\dagger}\right) \\
& \hat{y}_{\eta}=\frac{i}{\sqrt{2}}\left(o_{\eta}-o_{\eta}^{\dagger}\right)
\end{aligned}
$$

where $e_{\eta} / o_{\eta}$ are oscillators constructed with the twist even/odd part of the discrete spectrum eigenvectors $V_{N}^{(\bar{\xi})}, V_{N}^{(\xi)}$, see [16]

$$
\begin{gathered}
e_{\eta}=\sum_{N=0}^{\infty} \frac{1}{2}\left(1+(-1)^{N}\right) V_{N}^{(\xi)} a_{N} \\
o_{\eta}=\sum_{N=0}^{\infty} \frac{1}{2 i}\left(1-(-1)^{N}\right) V_{N}^{(\xi)} a_{N}
\end{gathered}
$$

The profile along these coordinates is given by (inverse Wick rotation, $(x, y) \rightarrow i(\mathrm{x},-\mathrm{y})$ is assumed)

$$
\left|\check{\Lambda}^{\prime}(\mathrm{x}, \mathrm{y})\right\rangle=\left\langle\mathrm{x}, \mathrm{y} \mid \check{\Lambda}^{\prime}\right\rangle=\frac{1}{\pi\left(1+e^{|\eta|}\right)} \exp \left(-\frac{e^{|\eta|}-1}{e^{|\eta|}+1}\left(\mathrm{x}^{2}+\mathrm{y}^{2}\right)\right)\left|\check{\Lambda}_{c}^{\prime}\right\rangle
$$

where $\left|\check{\Lambda}_{c}^{\prime}\right\rangle$ contains only continuous spectrum contributions. This profile is localized on the time coordinate $\mathrm{x}$. Note however that there is no more reference to the $E$-field in the exponent. In order to see explicitly the presence of the $E$-field, we need to use the usual open string time, i.e. the center of mass.

Therefore we contract our solution with the center of mass euclidean time, $x^{D}$, and then inverse Wick rotate it, $x^{D} \rightarrow i x^{0}$. This is identical to [16], so we just quote the result, paying attention to use the open string metric (2.2)

$$
\begin{aligned}
& \left|\Lambda^{\prime}\left(\mathrm{x}_{0}, y\right)\right\rangle=\left\langle\mathrm{x}_{0}, \mathrm{y} \mid \Xi_{\eta}\right\rangle=\sqrt{\frac{2}{b \pi}} \frac{\mathcal{N}}{\sqrt{2 \pi\left(1+e^{|\eta|}\right)}} \exp \left(\frac{1-e^{|\eta|}}{1+e^{|\eta|}} \mathrm{y}^{2}\right) \\
& \frac{1}{\sqrt{1+\check{T}_{00}^{\prime}}} \exp \left(-\mathcal{A}\left(x^{0}\right)^{2}+\frac{2 i \sqrt{1-(2 \pi E)^{2}}}{\sqrt{b}\left(1+\check{T}_{00}^{\prime}\right)} x^{0} \check{T}_{0 n}^{\prime} \tilde{a}_{n}^{\dagger}-\frac{1}{2} \tilde{a}_{n}^{\dagger} W_{n m}^{\prime \prime} \tilde{a}_{m}^{\dagger}\right)|0\rangle
\end{aligned}
$$

The extra coordinate $\mathrm{y}$ is given by the twist odd contribution of the discrete spectrum, we need to project along it in order to have a well defined $b \rightarrow \infty$ limit in the oscillator part $W_{n m}^{\prime \prime}$, see [16]. The oscillators $\tilde{a}_{n}$ are canonically normalized with respect the $\eta$-metric and are given by

$$
\tilde{a}_{n}=\sqrt{1-(2 \pi E)^{2}} a_{n}
$$

The quantity that give rise to time localization is then

$$
\mathcal{A}=-\frac{1}{b} \frac{1-\check{T}_{00}^{\prime}}{1+\check{T}_{00}^{\prime}}\left(1-(2 \pi E)^{2}\right)
$$


This quantity depends on the free parameter $b$, as well as on the value of the $E$-field, through the open string metric, used to covariantize the quadratic form in time. The matrix element $\check{T}_{00}^{\prime}$ is given in [16]

$$
\check{T}_{00}^{\prime}(\eta)=-2 \int_{0}^{\infty} d k\left(V_{0}^{(k)}(b(\eta))\right)^{2} \exp \left(-\frac{\pi k}{2}\right)+2\left(V_{0}^{(\xi)}\right)^{2} \exp |\eta|
$$

it is a monotonic increasing function of $b$, greater than 1: this is what ensures localization in time as opposed to the standard lump which is suited for space localization.

The life time of the brane is thus given by

$$
\Delta T=\frac{1}{2} \sqrt{\frac{1}{2 \mathcal{A}}}=\frac{1}{\left(1-(2 \pi E)^{2}\right)^{\frac{1}{2}}} \Delta T^{(E=0)}
$$

Note that for $E$ going to the critical value $E_{c}=\frac{1}{2 \pi}$, the lifetime becomes infinite. In particular we get a completely flat profile. This has to be traced back to the fact that open strings become effectively tensionless in this limit, [19], so we correctly recover the result of [16], that the D-brane is stable. This configuration should correspond to a background of fundamental strings stretched along the $E$-field direction, with closed strings completely decoupled.

\section{Transverse $E$-field}

In this section we study the time dynamics of a D-brane with transverse $E$-field. We will do this in two steps. First we will write down coordinates and momenta operators corresponding to the oscillators of the discrete diagonal basis and look at the profile of the lump solution with respect to them. Next we will determine the open string time profile of the lump solution by projecting it onto the center of mass coordinates. Since the solutions with $E$-field are equivalent to euclidean solutions with imaginary $B$-field, before proceeding further, we will first give a brief summary of the construction of lump solutions in VSFT with transverse $B$-field.

\subsection{Lump solutions with B field}

The solitonic lump solutions in VSFT in the presence of a constant transverse $B$ field were determined in 31]. The $*$ product is defined as follows

$$
{ }_{123}\left\langle V_{3} \mid \Psi_{1}\right\rangle_{1}\left|\Psi_{2}\right\rangle_{2}={ }_{3}\left\langle\Psi_{1} *_{m} \Psi_{2}\right|
$$

where the 3 -string vertex $V_{3}$, with a constant $B$ field turned on along the $24^{\text {th }}$ and $25^{\text {th }}$ directions (in view of the D-brane interpretation, these directions are referred to as transverse), is

$$
\left|V_{3}\right\rangle=\left|V_{3, \perp}\right\rangle \otimes\left|V_{3, \|}\right\rangle
$$

$\left|V_{3,||}\right\rangle$ corresponds to the tangential directions while $\left|V_{3, \perp}\right\rangle$ is obtained from [22] by passing to zero modes oscillator basis and integrating over transverse momenta, see [31]

$$
\left|V_{3, \perp}\right\rangle=\frac{\sqrt{2 \pi b^{3} \Delta}}{A^{2}\left(4 a^{2}+3\right)} \exp \left[\frac{1}{2} \sum_{r, s=1}^{3} \sum_{N, M \geq 0} a_{M}^{(r) \alpha \dagger} \mathcal{V}_{\alpha \beta, M N}^{r s} a_{N}^{(r) \beta \dagger}\right]|0\rangle \otimes\left|\Omega_{b, \theta}\right\rangle_{123}
$$


In the following we will set $\alpha, \beta=1,2$ for simplicity of notation. $\left|\Omega_{b, \theta}\right\rangle$ is the vacuum with respect to the zero mode oscillators

$$
a_{0}^{(r) \alpha}=\frac{1}{2} \sqrt{b} \hat{p}^{(r) \alpha}-i \frac{1}{\sqrt{b}} \hat{x}^{(r) \alpha}, \quad a_{0}^{(r) \alpha \dagger}=\frac{1}{2} \sqrt{b} \hat{p}^{(r) \alpha}+i \frac{1}{\sqrt{b}} \hat{x}^{(r) \alpha} .
$$

$\mathcal{V}_{\alpha \beta, M N}^{r s}$ are the Neumann coefficients with zero modes in a constant $B$ field background, which are symmetric under simultaneous exchange of all the three pairs of indices and cyclic in the string label indices $(r, s)$ where $r, s=4$ is identified with $r, s=1$. Moreover $\Delta=\sqrt{\operatorname{Det} G}, G_{\alpha \beta}$ being the open string metric along the transverse directions (2.2). We have also introduced the notations

$$
A=V_{00}+\frac{b}{2}, \quad a=-\frac{\pi^{2}}{A}|B| .
$$

The lump solution is given by

$$
|S\rangle=\left|S_{\|}\right\rangle \otimes \mathcal{N} \exp \left(-\frac{1}{2} \sum_{M, N \geq 0} a_{M}^{\alpha \dagger} \mathcal{S}_{\alpha \beta, M N} a_{N}^{\beta \dagger}\right)|0\rangle \otimes\left|\Omega_{b, \theta}\right\rangle,
$$

where

$$
\mathcal{N}=\frac{A^{2}\left(3+4 a^{2}\right)}{\sqrt{2 \pi b^{3}}(\operatorname{Det} G)^{\frac{1}{4}}} \operatorname{Det}(\mathcal{I}-\mathcal{X})^{\frac{1}{2}} \operatorname{Det}(\mathcal{I}+\mathcal{T})^{\frac{1}{2}}
$$

and

$$
\mathcal{X}=C^{\prime} \mathcal{V}^{11}, \quad \mathcal{T}=C^{\prime} \mathcal{S}, \quad C^{\prime}=(-1)^{N} \delta_{N M}
$$

In (3.6) $\left|S_{\|}\right\rangle$corresponds to the longitudinal part of the lump solution and it is a zero momentum sliver.

In order for (3.6) to satisfy the projector equation, $\mathcal{T}$ and $\mathcal{X}$ should satisfy the relation ${ }^{8}$

$$
(\mathcal{T}-1)\left(\mathcal{X} \mathcal{T}^{2}-(\mathcal{I}+\mathcal{X}) \mathcal{T}+\mathcal{X}\right)=0
$$

In the above formulae the $\alpha, \beta, N, M$ indices are implicit. This equation is solved by $\mathcal{T}_{0}$, $1 / \mathcal{T}_{0}$ and 1 , where

$$
\mathcal{T}_{0}=\frac{1}{2 \mathcal{X}}(1+\mathcal{X}-\sqrt{(1+3 \mathcal{X})(1-\mathcal{X})})
$$

$\mathcal{T}=1$ gives the identity state, whereas the first and the second solutions give the lump and the inverse lump, respectively. In [16] it has been argued that, although the inverse lump solution was discarded in earlier works [29, 30], because of the bad behaviour of its eigenvalues in the oscillator basis, it is possible to make sense out of it by considering (3.9) as a relation between eigenvalues relative to twist definite eigenvectors. In particular, in the diagonal basis, the projector equation factorizes into the continuous and discrete contributions, which separately satisfy equation (3.9). Therefore, one can just invert (for example) the discrete eigenvalues of $\mathcal{T}$ : dangerous - signs under the square root in the energy densities of the solution are indeed avoided by counting the double multiplicity of these eigenvalues, which is required by twist invariance. See Appendix A for the spectroscopy of $\mathcal{X}$, and hence of $\mathcal{T}$.

\footnotetext{
${ }^{8}$ In this paper we limit ourselves to twist invariant projectors, but our analysis can be straightforwardly generalized to projectors of the kind [24]
} 


\subsection{Diagonal Coordinates and Momenta}

In Appendix B $\tau$-twist definite oscillators of the diagonal basis are introduced. Due to the structure of Neumann coefficients it is natural to define the twist matrix as $\tau C$, where $\tau=\sigma^{3}$ acts on space-time indices, see appendices $\mathrm{A}$ and $\mathrm{B}$ for details. In the following $C$-parity will be always understood as $\tau C$-parity. Now let's define the following coordinates and momenta operators in terms of the twist even and twist odd parts of the discrete spectrum, (B.6)

$$
\hat{X}_{\xi_{i}}=\frac{i}{\sqrt{2}}\left(e_{\xi_{i}}-e_{\xi_{i}}^{\dagger}\right) \quad \hat{Y}_{\xi_{i}}=\frac{i}{\sqrt{2}}\left(o_{\xi_{i}}-o_{\xi_{i}}^{\dagger}\right)
$$

which are hermitian by definition and have the following eigenstates

$$
\begin{aligned}
& \left|X_{i}\right\rangle=\frac{1}{\sqrt{\pi}} e^{-\frac{1}{2} X_{i}^{2}-\sqrt{2} i X_{i} e_{\xi_{i}}^{\dagger}+\frac{1}{2} e_{\xi_{i}}^{\dagger} e_{\xi_{i}}^{\dagger}\left|\Omega_{e_{i}}\right\rangle} \\
& \left|Y_{i}\right\rangle=\frac{1}{\sqrt{\pi}} e^{-\frac{1}{2} Y_{i}^{2}-\sqrt{2} i Y_{i} o_{\xi_{i}}^{\dagger}+\frac{1}{2} o_{\xi_{i}}^{\dagger} o_{\xi_{i}}^{\dagger}}\left|\Omega_{O_{i}}\right\rangle .
\end{aligned}
$$

We made the assumption that the vacuum factorizes as

$$
|0\rangle \otimes\left|\Omega_{b, \theta}\right\rangle=\prod_{i=1}^{2} \prod_{k}\left|\Omega_{i}(k)\right\rangle \otimes\left|\Omega_{e_{i}}\right\rangle \otimes\left|\Omega_{o_{i}}\right\rangle
$$

where $\left|\Omega_{i}(k)\right\rangle,\left|\Omega_{e_{i}}\right\rangle$ and $\left|\Omega_{o_{i}}\right\rangle$ are vacua with respect to the continuous, the twist even discrete and twist odd discrete oscillators, respectively.

The explicit $\left(X_{i}, Y_{i}\right)$ dependence of the lump state (3.6) can be obtained by projecting it onto the eigenstates $\left|X_{i}, Y_{i}\right\rangle$. After re-writing (3.6) in terms of the diagonal basis oscillators and performing the projection (see Appendix B), it follows

$$
\begin{array}{r}
\left\langle X_{i}, Y_{i} \mid S\right\rangle=\frac{1}{\pi^{2}\left[1+t_{d}\left(\eta_{1}\right)\right]\left[1+t_{d}\left(\eta_{2}\right)\right]} \exp \frac{1}{2}\left[\frac{t_{d}\left(\eta_{1}\right)-1}{t_{d}\left(\eta_{1}\right)+1}\left(X_{1}^{2}+Y_{1}^{2}\right)\right. \\
\left.+\frac{t_{d}\left(\eta_{2}\right)-1}{t_{d}\left(\eta_{2}\right)+1}\left(X_{2}^{2}+Y_{2}^{2}\right)\right]|S\rangle_{c} \otimes\left|S_{\|}\right\rangle .
\end{array}
$$

$|S\rangle_{c}$ is given by (B.11) with only continuous spectrum oscillators and $t_{d}\left(\eta_{i}\right)=e^{-\left|\eta_{i}\right|}$ are the discrete eigenvalues of $\mathcal{T}$ corresponding to the eigenvalue $\xi\left(\eta_{i}\right)$ of the operator $C^{\prime} \mathcal{U}$.

In (3.15) the directions $\alpha, \beta$ are completely mixed. As a matter of fact, it is not apparent at this stage which of these variables $\left(X_{i}, Y_{i}\right)$ contain the information about the center of mass time dependence of the lump. To make this clear let's recall the non-diagonal basis oscillators and write the coordinates and the momenta operators as

$$
\hat{X}_{N}^{\alpha}=\frac{i}{\sqrt{2}}\left(a_{N}^{\alpha}-a_{N}^{\alpha \dagger}\right) \quad \hat{P}_{N}^{\alpha}=\frac{1}{\sqrt{2}}\left(a_{N}^{\alpha}+a_{N}^{\alpha \dagger}\right) .
$$

In order to get the relation between these operators and the corresponding diagonal operators we have defined above, we need to re-write the diagonal basis oscillators in terms of the non-diagonal ones. In doing so, one has to be careful about taking the complex conjugate of 
the eigenstates, as we are dealing with hermitian rather then symmetric matrices. Taking this fact into account and using some results of Appendix B, we obtain

$$
\begin{array}{ll}
e_{\xi_{i}}=\frac{1}{\sqrt{2}} \sum_{N=0}^{\infty}\left(V_{N}^{\left(\xi_{i}\right) \alpha}+V_{N}^{\left(\bar{\xi}_{i}\right) \alpha}\right) a_{N, \alpha} & e_{\xi_{i}}^{\dagger}=\frac{1}{\sqrt{2}} \sum_{N=0}^{\infty}\left(\bar{V}_{N}^{\left(\xi_{i}\right) \alpha}+\bar{V}_{N}^{\left(\bar{\xi}_{i}\right) \alpha}\right) a_{N, \alpha}^{\dagger} \\
o_{\xi_{i}}=\frac{-i}{\sqrt{2}} \sum_{N=0}^{\infty}\left(V_{N}^{\left(\xi_{i}\right) \alpha}-V_{N}^{\left(\bar{\xi}_{i}\right) \alpha}\right) a_{N, \alpha} & o_{\xi_{i}}^{\dagger}=\frac{i}{\sqrt{2}} \sum_{N=0}^{\infty}\left(\bar{V}_{N}^{\left(\xi_{i}\right) \alpha}-\bar{V}_{N}^{\left(\bar{\xi}_{i}\right) \alpha}\right) a_{N, \alpha}^{\dagger}
\end{array}
$$

and similar relations for the continuous spectrum oscillators. Hence, the diagonal coordinates and momenta can be written as

$$
\begin{aligned}
& \hat{X}_{\xi_{i}}=\sqrt{2} \sum_{N=0}^{\infty} V_{2 N}^{\xi_{i}, 1} \hat{X}_{2 N}^{1}+V_{2 N+1}^{\xi_{i}, 2} \hat{P}_{2 N+1}^{2} \\
& \hat{Y}_{\xi_{i}}=\sqrt{2} \sum_{N=0}^{\infty} V_{2 N+1}^{\xi_{i}, 1} \hat{P}_{2 N+1}^{1}-i V_{2 N}^{\xi_{i}, 2} \hat{X}_{2 N}^{2}
\end{aligned}
$$

Now, to make the center of mass time dependence of the solution explicit, we need to extract the zero modes from these operators. Let's write the zero mode coordinate and momentum operators by introducing the b parameter as

$$
\hat{X}_{0}^{\alpha}=\frac{i}{\sqrt{b}}\left(a_{0}^{\alpha}-a_{0}^{\alpha \dagger}\right) \quad \hat{P}_{0}^{\alpha}=\frac{\sqrt{b}}{2}\left(a_{0}^{\alpha}+a_{0}^{\alpha \dagger}\right) .
$$

This gives

$$
\begin{aligned}
& \hat{X}_{\xi_{i}}=\sqrt{2}\left[V_{0}^{\xi_{i}, 1} \sqrt{\frac{2}{b}} X_{0}^{1}+\sum_{n=1}^{\infty} V_{2 n}^{\xi_{i}, 1} \hat{X}_{2 n}^{1}+V_{2 n-1}^{\xi_{i}, 2} \hat{P}_{2 n-1}^{2}\right], \\
& \hat{Y}_{\xi_{i}}=\sqrt{2}\left[V_{0}^{\xi_{i}, 2} \sqrt{\frac{2}{b}} X_{0}^{2}+\sum_{n=1}^{\infty} V_{2 n-1}^{\xi_{i}, 1} \hat{P}_{2 n-1}^{1}-i V_{2 n}^{\xi_{i}, 2} \hat{X}_{2 n}^{2}\right] .
\end{aligned}
$$

Since our aim is to obtain the localization in time by making the inverse Wick rotation on direction 1, we see that it is $X_{\xi_{i}}$ that contains the time coordinate, which we have to compare with the string center of mass time (see below).

\subsection{Projection on the center of mass coordinates}

In order to obtain the open string time profile of the lump solution, we need to project it onto the center of mass coordinates of the string. The center of mass position operator is given by

$$
\hat{x}_{c m, \alpha}=\frac{i}{\sqrt{b}}\left(a_{0, \alpha}-a_{0, \alpha}^{\dagger}\right)
$$

and its eigenstate is

$$
\left|X_{C M}\right\rangle=\sqrt{\frac{2 \Delta}{\pi b}} e^{-\frac{1}{b} x_{\alpha} x^{\alpha}-\frac{2}{\sqrt{b}} i x_{\alpha} a_{0}^{\alpha \dagger}+\frac{1}{2} a_{0, \alpha}^{\dagger} a_{0}^{\alpha \dagger}}\left|\Omega_{\theta, b}\right\rangle .
$$


One can project the lump on this state to obtain the center of mass time profile. However, for reasons that will be clear later, we will first project on the $Y_{i}$ momenta,

$$
\begin{aligned}
& |\Lambda\rangle=\left\langle Y_{1}, Y_{2} \mid S\right\rangle=\frac{\mathcal{N}}{\pi \sqrt{\left[1+t_{d}\left(\eta_{1}\right)\right]\left[1+t_{d}\left(\eta_{2}\right)\right]}} \exp \frac{1}{2}\left[\frac{t_{d}\left(\eta_{1}\right)-1}{t_{d}\left(\eta_{1}\right)+1} Y_{1}^{2}+\frac{t_{d}\left(\eta_{2}\right)-1}{t_{d}\left(\eta_{2}\right)+1} Y_{2}^{2}\right] \\
& \quad \times \exp -\frac{1}{2}\left[e_{\xi_{i}}^{\dagger} e_{\xi_{i}}^{\dagger} t_{d}\left(\eta_{i}\right)+\int_{-\infty}^{\infty} d k a_{i}^{\dagger}(k) a_{i+1}^{\dagger}(-k) t_{c}(k)\right]\left|\Omega_{e}\right\rangle \otimes\left|\Omega_{c}\right\rangle \otimes\left|S_{\|}\right\rangle .
\end{aligned}
$$

Where we have used the notation

$$
\left|\Omega_{e}\right\rangle=\prod_{i=1}^{2}\left|\Omega_{e_{i}}\right\rangle, \quad\left|\Omega_{c}\right\rangle=\prod_{i=1}^{2} \prod_{k}\left|\Omega_{i}(k)\right\rangle .
$$

Taking equation (3.18) and the corresponding relations for the continuous spectrum oscillators, equation (3.26) can be rewritten as

$$
\begin{aligned}
|\Lambda\rangle & =\frac{\mathcal{N}}{\pi \sqrt{\left[1+t_{d}\left(\eta_{1}\right)\right]\left[1+t_{d}\left(\eta_{2}\right)\right]}} \exp \frac{1}{2}\left[\frac{t_{d}\left(\eta_{1}\right)-1}{t_{d}\left(\eta_{1}\right)+1} Y_{1}^{2}+\frac{t_{d}\left(\eta_{2}\right)-1}{t_{d}\left(\eta_{2}\right)+1} Y_{2}^{2}\right] \\
& \times \exp \left[-\frac{1}{2} a_{0, \alpha}^{\dagger} \hat{S}_{00}^{\alpha \beta} a_{0, \beta}^{\dagger}-a_{0, \alpha}^{\dagger} S_{0}^{\alpha}-\frac{1}{2} a_{n, \alpha}^{\dagger} \hat{S}_{n m}^{\alpha \beta} a_{m, \beta}^{\dagger}\right]\left|\hat{\Omega}_{b, \theta}\right\rangle \otimes\left|S_{\|}\right\rangle,
\end{aligned}
$$

where $\left|\hat{\Omega}_{b, \theta}\right\rangle=\left|\Omega_{e}\right\rangle \otimes\left|\Omega_{c}\right\rangle$ and

$$
\begin{gathered}
\hat{S}_{00}^{\alpha \beta}=\sum_{i=1}^{2} V_{0}^{\left(\xi_{i}^{+}\right) \alpha} \bar{V}_{0}^{\left(\xi_{i}^{+}\right) \beta} t_{d}\left(\eta_{i}\right)+\int_{-\infty}^{\infty} d k t(k) V_{0}^{i, \alpha}(k) \bar{V}_{0}^{i, \beta}(k) \\
S_{0}^{\alpha}=\sum_{i=1}^{2} \sum_{n=1}\left[V_{0}^{\left(\xi_{i}^{+}\right) \alpha} \bar{V}_{n}^{\left(\xi_{i}^{+}\right) \beta} t_{d}\left(\eta_{i}\right)+\int_{-\infty}^{\infty} d k t(k) V_{0}^{i, \alpha}(k) \bar{V}_{n}^{i, \beta}(k)\right] a_{n, \beta}^{\dagger}=\hat{S}_{0 n}^{\alpha \beta} a_{n, \beta}^{\dagger} \\
\hat{S}_{n m}^{\alpha \beta}=\sum_{i=1}^{2}(-1)^{n} V_{n}^{\left(\xi_{i}^{+}\right) \alpha} \bar{V}_{m}^{\left(\xi_{i}^{+}\right) \beta} t_{d}\left(\eta_{i}\right)+\int_{-\infty}^{\infty} d k t(k)(-1)^{n} V_{n}^{i, \alpha}(k) \bar{V}_{m}^{i, \beta}(k)
\end{gathered}
$$

with $V_{N}^{\left(\xi_{i}^{+}\right) \alpha}$ being the twist even combination of the discrete eigenstates, see appendix B. Now let's project onto the center of mass coordinates

$$
\begin{gathered}
\left\langle X_{C M} \mid \Lambda\right\rangle=\frac{\mathcal{N}}{\pi \sqrt{\left[1+t_{d}\left(\eta_{1}\right)\right]\left[1+t_{d}\left(\eta_{2}\right)\right]}} \exp \frac{1}{2}\left[\frac{t_{d}\left(\eta_{1}\right)-1}{t_{d}\left(\eta_{1}\right)+1} Y_{1}^{2}+\frac{t_{d}\left(\eta_{2}\right)-1}{t_{d}\left(\eta_{2}\right)+1} Y_{2}^{2}\right] \\
\times \sqrt{\frac{2 \Delta}{\pi b}} \frac{1}{\sqrt{\left[1+s_{1}\right]\left[1+s_{2}\right]}} \exp \frac{1}{b}\left[\frac{s_{1}-1}{s_{1}+1} x_{1} x^{1}+\frac{s_{2}-1}{s_{2}+1} x_{2} x^{2}+2 i \sqrt{b}\left(\frac{S_{0,1} x^{1}}{1+s_{1}}+\frac{S_{0,2} x^{2}}{1+s_{2}}\right)\right] \\
\times \exp \left[-\frac{1}{2} a_{n, \beta}^{\dagger}\left(\hat{S}_{n m}^{\alpha \beta}-\frac{\hat{S}_{n 0,1}^{\alpha} \hat{S}_{0 m}^{1 \beta}}{1+s_{1}}-\frac{\hat{S}_{n 0,2}^{\alpha} \hat{S}_{0 m}^{2 \beta}}{1+s_{2}}\right) a_{n, \beta}^{\dagger}\right]|0\rangle \otimes\left|S_{\|}\right\rangle
\end{gathered}
$$


where

$$
\begin{gathered}
s_{1}=2 \Delta\left[g_{d}^{2}\left(\eta_{1}, \eta_{2}\right) t_{d}\left(\eta_{1}\right)+g_{d}^{2}\left(\eta_{2}, \eta_{1}\right) t_{d}\left(\eta_{2}\right)\right]+\Delta \int_{-\infty}^{\infty} d k t_{c}(k)\left[g_{c}^{2}(k)+g_{c}^{2}(-k)\right), \\
s_{2}=\Delta \int_{-\infty}^{\infty} d k t_{c}(k)\left[g_{c}^{2}(k)+g_{c}^{2}(-k)\right]
\end{gathered}
$$

$t_{c}(k)=-e^{-\pi|k| / 2}$ is the eigenvalue of $\mathcal{T}$ in the continuous spectrum, see Appendix A for the definition of the remaining terms which enter in the last two equations. The inverse Wickrotation along direction 1 of (3.32) should give us a time-localized solution. It depends on two parameters, $b$ and $a$, which can be expressed in terms of $\left(\eta_{1}, \eta_{2}\right)$, through the eigenvalues equations A.16 . Let's now take a look at every term in this solution and analyze it for different values of the such parameters.

In the Wick-rotated solution, to get time-localization, the term $-\frac{1}{b} \frac{s_{1}-1}{s_{1}+1}$ should be negative. We cannot achieve this using the conventional lump, since in this case $-1<s_{1}<1$. To correct this, as anticipated, we need to invert one or two discrete eigenvalues, $\left(t_{d}\left(\eta_{1}\right)\right.$ or/and $\left.t_{d}\left(\eta_{2}\right)\right)$. In this case one can easily show that $1<s_{1}<\infty$ and we get the desired behaviour. Given the possibility of inverting one or two eigenvalues, it might seem that there is some arbitrariness in our procedure. Actually there is none, since the cancelation of the potentially divergent terms when $b \rightarrow \infty$ (see below), requires the inversion of only one eigenvalue. In addition, time localization in small $b$ regime requires the inversion of the eigenvalue of $\mathcal{T}$ corresponding to the greater between $\eta_{1}$ and $\eta_{2}$ ( $\eta_{2}$ in our conventions). From now on we will then consider a solution in which $t_{d}\left(\eta_{2}\right)$ is inverted, i.e. $t_{d}\left(\eta_{2}\right) \rightarrow t_{d}^{-1}\left(\eta_{2}\right)$.

Next, look at the term $\frac{s_{2}-1}{s_{2}+1} x_{2} x^{2}$. Due to the $\left\langle Y_{1}, Y_{2}\right|$ projection, it gets a contribution only from the continuous spectrum, which is always negative and in the range $(-1,0)$. As a result, this second term is always negative and gives localization in the transverse space direction.

Now we would like to point out some facts about the two parameters on which our solution depends. In [16], it has been pointed out that the inverse of the parameter $b$, for large $b$, plays the role of Sen's $\tilde{\lambda}$ near zero. Here again we can repeat the same argument. Note however that in taking $b$ to infinity we should keep $a$ vanishing, see (3.5), since we cannot overcome the critical value $|B|_{c}=\frac{1}{2 \pi}$. For this reason the result of taking $b \rightarrow \infty$ is insensible of the value of the $E$-field, making this limit completely commutative.

As it is justified in Appendix $\mathrm{C}$, the proper way to send $b$ to $\infty$ is to take $\eta_{1} \approx \eta_{2} \rightarrow \infty$ keeping $\eta_{1}<\eta_{2}$. In this case one can easily see that

$$
s_{1} \approx \eta_{2} \log \eta_{1} \eta_{2}+t_{c}\left(k_{0} \approx 0\right), \quad s_{2} \approx t_{c}\left(k_{0} \approx 0\right)
$$

with $k_{0}$ as defined in Appendix C. Note that $t_{c}\left(k_{0} \approx 0\right)=-1$. This is so because the $E$-field cannot scale to infinity due to existence of critical value. Then it follows

$$
\lim _{\eta_{1}, \eta_{2} \rightarrow \infty} \frac{s_{1}-1}{s_{1}+1}=1, \quad \lim _{\eta_{1}, \eta_{2} \rightarrow \infty} \frac{s_{2}-1}{s_{2}+1}=-\infty
$$

As justified in Appendix C.1.2, in this limit $\hat{S}_{n 0}^{\alpha \beta(c)}=0$ so that the oscillating term in (3.32) receives a contribution only from the discrete part. It is also pointed out that the discrete 
contribution vanishes except for $\alpha=\beta=1$, which is the only non trivial contribution to the oscillating term. Moreover, we have

$$
\lim _{\eta_{1}, \eta_{2} \rightarrow \infty} \frac{\Delta \hat{S}_{n 0}^{11}}{s_{1}+1}=(-1)^{n} \lim _{\eta_{1}, \eta_{2} \rightarrow \infty} \frac{1}{2 \sqrt{\log \eta_{1} \eta_{2}}}=0,
$$

Therefore, the oscillating term in (3.32) vanishes when $b \rightarrow \infty$.

Now let's consider the non-zero mode terms, i.e, the last line in (3.32). In the $b \rightarrow \infty$ limit it is clear that $V_{n}^{\left(\xi_{i}^{+}\right) \alpha}$ vanishes for $\alpha=2$ and $n \geq 1$. Therefore, the contribution of the discrete spectrum to $\hat{S}_{n m}^{\alpha \beta}$ is zero for $\alpha$ or $\beta=2$ and $n, m \geq 1$. However, for $\alpha=\beta=1$ this is not true and there are potentially divergent contributions from the discrete spectrum. We are now going to show that these divergences cancel and the expression

$$
\check{S}_{n m}^{11}=\hat{S}_{n m}^{11(c)}+\hat{S}_{n m}^{11(d)}-\frac{\hat{S}_{n 0,1}^{1(d)} \hat{S}_{0 m}^{11(d)}}{1+s_{1}} .
$$

is finite when $b \rightarrow \infty$.

To this end we notice that, inverting only $t_{d}\left(\eta_{2}\right)$ but taking both $\eta_{1}$ and $\eta_{2}$ to infinity, the different terms which enter in the above expression have the following behaviours

$$
\begin{gathered}
1+s_{1} \approx \Delta t_{d}^{-1}\left(\eta_{2}\right) V_{0}^{\left(\xi_{2}^{+}\right), 1} \bar{V}_{0}^{\left(\xi_{2}^{+}\right), 1}, \\
\hat{S}_{n 0,1}^{1(d)} \approx \Delta(-1)^{n} t_{d}^{-1}\left(\eta_{2}\right) V_{n}^{\left(\xi_{2}^{+}\right), 1} \bar{V}_{0}^{\left(\xi_{2}^{+}\right), 1}, \\
\hat{S}_{n 0}^{11(d)} \approx(-1)^{n} t_{d}^{-1}\left(\eta_{2}\right) V_{n}^{\left(\xi_{2}^{+}\right), 1} \bar{V}_{0}^{\left(\xi_{2}^{+}\right), 1}, \\
\hat{S}_{n m}^{11(d)} \approx(-1)^{n} t_{d}^{-1}\left(\eta_{2}\right) V_{n}^{\left(\xi_{2}^{+}\right), 1} \bar{V}_{m}^{\left(\xi_{2}^{+}\right), 1},
\end{gathered}
$$

Note that $t_{d}^{-1}\left(\eta_{2}\right)=e^{\left|\eta_{2}\right|}$ gives a divergent contribution as $\eta_{2} \rightarrow \infty$. However, using these results in eq.(3.37), it is easy to see that the divergent terms cancel and we are left with $\check{S}_{n m}^{11}=\hat{S}_{n m}^{11(c)}$. This, combined with the fact that for $\alpha=2$ or $\beta=2$ we have $\hat{S}_{N M}^{\alpha \beta(d)}=0$, leads us to the conclusion that $\check{S}_{n m}^{\alpha \beta}=\hat{S}_{n m}^{\alpha \beta(c)}+O\left(\frac{1}{b}\right)$. It is also verified in Appendix C that $\hat{S}_{n m}^{11(c)}=\hat{S}_{n m}^{22(c)}=S_{n m}$.

This also show that is not possible to invert both the discrete eigenvalues and obtain the same cancelation. Indeed, if we invert both, the term $\hat{S}_{n 0,1}^{1(d)} \hat{S}_{0 m}^{11(d)}$ contains mixed terms like $\left[t^{-1}\left(\eta_{1}\right) V_{n}^{\left(\xi_{1}^{+}\right), 1} \bar{V}_{0}^{\left(\xi_{1}^{+}\right), 1}\right]\left[t^{-1}\left(\eta_{2}\right) V_{n}^{\left(\xi_{2}^{+}\right), 1} \bar{V}_{0}^{\left(\xi_{2}^{+}\right), 1}\right]$, for which we cannot find a counter term in $\hat{S}_{n m}^{11(d)}$ to cancel it. As a result we will not be able to get a regular time and space localized solution, since these terms diverge in the limit $\eta_{1}, \eta_{2} \rightarrow \infty$.

After all these remarks, we can write the space-time localized solution in the $b \rightarrow \infty$ limit as

$$
\lim _{b \rightarrow \infty}\left\langle X_{C M} \mid \Lambda\right\rangle_{W i c k}=N\left(Y_{1}, Y_{2}\right) \lim _{b \rightarrow \infty} e^{-\frac{\Delta}{b}\left(x^{0}\right)^{2}} e^{-\epsilon(b)\left(x^{2}\right)^{2}}|S\rangle
$$

where $|S\rangle$ is the space-time independent VSFT solution (the sliver). Note that time dependence completely disappears in this limit . A remark is in order for the quantity $\epsilon(b)$ this number is given by, see (3.32)

$$
\epsilon(b)=\frac{\Delta}{b} \frac{s_{2}-1}{s_{2}+1}
$$


a numerical analysis shows that this becomes vanishing as $b \rightarrow \infty$. One can indeed easily check (numerically) that the $\frac{1}{b}$ correction to $\frac{s_{2}(b)-1}{s_{2}(b)+1}$ diverges. This in turn implies that the loss of time dependence is accompanied by loss of transverse space dependence, giving a resulting zero momentum state (the D25-sliver). Therefore, taking $b$ to infinity is like sitting at the original unstable vacuum (the D25-brane), which is the same situation as setting Sen's $\tilde{\lambda}$ to zero.

Another remark we would like to make is about small $b$ limit, which we can get by taking $\eta_{1} \rightarrow 0$ and keeping $\eta_{2}$ finite. Given that the large $b$ limit corresponds to Sen's $\tilde{\lambda}$ near zero (i.e it represents the unstable vacuum), it is natural to think that the small $b$ limit corresponds to $\tilde{\lambda}$ near $\frac{1}{2}$ (or the stable vacuum). As a matter of fact, taking this limit of $b$ one gets the 0 state, which is also obtained in the $x_{0} \rightarrow \infty$ limit and corresponds to the stable vacuum to which the D-brane decays. This can be seen by noting that, in this case, $V_{0}^{\alpha}(k) \rightarrow 0$, whereas $V_{n}^{\alpha}(k)$ for $n \geq 1$ have a finite nonvanishing limit. As a result $s_{1}$ do not get a contribution from the continuous spectrum and $s_{2}=0$. Then, it follows

$$
-\frac{\Delta}{b} \frac{s_{1}-1}{s_{1}+1} \approx-\frac{\Delta}{\eta_{1}}\left(\left|\frac{s_{1}-1}{s_{1}+1}\right|\right), \quad \frac{\Delta}{b} \frac{s_{2}-1}{s_{2}+1} \approx-\frac{\Delta}{\eta_{1}}
$$

where we have used $\left(b \approx \eta_{1}\right),\left(s_{1} \approx 1+O\left(\sqrt{\eta}_{1}\right)\right)$ in the limit $\eta_{1} \rightarrow 0$ and $\eta_{2}$ finite. These are results one can easily obtain from appendix C.2. For $\Delta \neq 0$ both of these terms gives a negative infinity in the exponent and suppress everything in front to give us the 0 state which corresponds to the stable vacuum. However, the case $\Delta=0$ should be handled with care. In this case, one can send $\Delta$ and $\eta_{1}$ to zero, in such a way that the ratio $\frac{\Delta}{\eta_{1}}$ remains finite. As a result the time dependence will be lost while the solution is still space localized. One should compare this with the time independent solution obtained when we send Sen's $\tilde{\lambda}$ to $\frac{1}{2}$ and, at the same time, tune the $E$-field to its critical value, [17, obtaining a static fundamental strings background.

\section{Conclusions}

In this work we have shown that the $E$-field deformed star algebra still presents time localized idempotents, which are a generalization of their $E=0$ cousins.

We have analyzed in detail the case of a longitudinal E-field (D25-brane decay) and of a transverse one (D24-brane decay).

In both cases time localization is achieved by working in the Wick rotated euclidean theory with an unconventional lump having inverted discrete eigenvalues of the Neumann matrix with respect to the conventional one. In particular the decaying D-24 brane solution has the nice feature of being localized in time and on the 25-th spatial direction (although for the extreme case $b \rightarrow \infty$ the transverse space profile becomes flat). This is a consequence of the fact that we first projected the unconventional lump on the twist odd momenta of the discrete spectrum and then on the center of mass coordinates, haven't we done this we would have lost space localization in the 24-th direction.

Our solution depends on two parameter, $b$ and $E$. After projecting on the center of mass time coordinate we have shown that in the extreme cases $b=0$ and $b=\infty$ time dependence is lost and we are left respectively with the 0 state (VSFT vacuum) and the 
sliver state (VSFT D-brane), confirming an earlier hypothesis, 16, that, although a finite value of $b$ can be formally changed by a gauge transformation, 30, these two cases, at least qualitatively, seems to reproduce the $\tilde{\lambda}=\frac{1}{2}$ and $\tilde{\lambda}=0$ of Sen's BCFT, 25. The $E$-field is another free parameter in the range $0 \leq E \leq E_{c}=\frac{1}{2 \pi \alpha^{\prime}}$. For $E$ going to its critical value, time dependence is lost and we get a flat (non zero) profile in time (effective tensionless regime), this result still persists in the $b \rightarrow 0$ limit if we keep the ratio $\frac{\Delta}{b}$ finite. This case should correspond to the tachyon vacuum with a background of fundamental strings, prevented to decay by their polarization due to critical electric field. Of course this is a very indirect way to see these fundamental strings arising in a classical solution of VSFT, a more direct construction of such objects is given in [18].

What we think is pressing, at the moment, is some workable way to define the energy momentum tensor for time dependent (V)SFT solutions, possibly along the lines of [32]. It would allow to extract physical informations, first of all their energy. This in turn would prove very useful in understanding how energy is conserved, while open string degrees of freedom are suppressed (except the $E$-field charged fundamental strings) and hence how open/closed duality is implemented, 25, 26, 27, 28,

\section{Acknowledgments}

We thank L. Bonora for collaboration and useful discussions at various stages of the work. C.M. thanks G. Bonelli and M. Salizzoni for discussions. This research was supported by the Italian MIUR under the program "Teoria dei Campi, Superstringhe e Gravità" and by CAPES-Brasil, as far as R.J.S.S. is concerned.

\section{Appendices}

\section{A Spectroscopy of the Neumann matrices with B field}

In this appendix we present the computation of the eigenstates and eigenvalues of the Neumann matrix $\mathcal{X}_{\beta}^{\alpha}$ in the presence of $B$-field, along the line of [21]. A similar analysis was carried out in 20], but with no reference to the correct normalization of continuous and discrete eigenvectors; moreover the discrete eigenvectors presented in the first of [20] does not reproduce the known ones when $B \rightarrow 0$. Since the discrete spectrum is of crucial importance for our purposes we re-derive completely the whole spectroscopy taking care of the correct normalization of continuous and discrete eigenvalues, as in 21. To avoid the degeneracy of the diagonal Neumann coefficient $\mathcal{X}_{\beta}^{\alpha}$, we consider the unitary matrices $C^{\prime} \mathcal{U}_{\beta}^{\alpha}$ and $\mathcal{U}_{\beta}^{\alpha} C^{\prime}$, which are related to $\mathcal{X}^{\alpha}{ }_{\beta}$ as follows 31]

$$
\left(\mathcal{X}_{\beta}^{\alpha}\right)_{N M}=\frac{1}{3}\left(\delta^{\alpha}{ }_{\beta}+C^{\prime} \mathcal{U}_{\beta}^{\alpha}+C^{\prime} \overline{\mathcal{U}}_{\beta}^{\alpha}\right)_{N M} .
$$

The matrix $\left(C^{\prime} \mathcal{U}_{\beta}^{\alpha}\right)_{N M}$ can be written explicitly as

$$
C^{\prime} \mathcal{U}=\left(\begin{array}{cccc}
1-3 b K & 2 \sqrt{3} b K a & 3 \sqrt{b} K\langle W| & -2 \sqrt{3 b} K a\langle W| \\
-2 \sqrt{3} b K a & 1-3 b K & 2 \sqrt{3 b} K a\langle W| & 3 \sqrt{b} K\langle W| \\
3 \sqrt{b} K|W\rangle & -2 \sqrt{3 b} K a|W\rangle & C U-3 K|W\rangle\langle W| & 2 \sqrt{3} K a|W\rangle\langle W| \\
2 \sqrt{3 b} K a|W\rangle & 3 \sqrt{b} K|W\rangle & -2 \sqrt{3} K a|W\rangle\langle W| & C U-3 K|W\rangle\langle W|
\end{array}\right)
$$


where, see [16]

$$
|W\rangle=-\sqrt{2}\left(\left|v_{e}\right\rangle+i\left|v_{o}\right\rangle\right), \quad K=\frac{A^{-1}}{4 a^{2}+3} .
$$

$C U$ is the non-zero mode analog of $C^{\prime} \mathcal{U}$ without $B$ field. We recall that, 31,

$$
C^{\prime} \overline{\mathcal{U}}=\tilde{\mathcal{U}} C^{\prime}
$$

where tilde means transposition with respect to $\alpha, \beta$ indices.

Our aim is to solve the eigenvalue equation

$$
C^{\prime} \mathcal{U}|\Psi\rangle=\xi|\Psi\rangle, \quad|\Psi\rangle=\left(\begin{array}{c}
g_{1} \\
g_{2} \\
\left|\Lambda_{1}\right\rangle \\
\left|\Lambda_{2}\right\rangle
\end{array}\right)
$$

which splits into

$$
\begin{gathered}
\left\langle W \mid \Lambda_{1}\right\rangle=\frac{A}{\sqrt{b}}\left[\xi-1+\frac{b}{A}\right] g_{1}+\frac{2 A a}{\sqrt{3 b}}(\xi-1) g_{2} \\
\left\langle W \mid \Lambda_{2}\right\rangle=\frac{A}{\sqrt{b}}\left[\xi-1+\frac{b}{A}\right] g_{2}-\frac{2 A a}{\sqrt{3 b}}(\xi-1) g_{1} \\
(C U-\xi)\left|\Lambda_{1}\right\rangle=\sqrt{\frac{1}{b}} g_{1}(\xi-1)|W\rangle \\
(C U-\xi)\left|\Lambda_{2}\right\rangle=\sqrt{\frac{1}{b}} g_{2}(\xi-1)|W\rangle
\end{gathered}
$$

We know, 33, that $C U$ has a continuous spectrum and the solution of (A.4) depends on whether the eigenvalue $\xi$ is in the continuous spectrum of $C U$ or not. So we will distinguish these two different cases and analyze each of them in detail.

\section{A.1 Discrete spectrum}

If $\xi$ is not in the spectrum of $C U$, we can invert $(C U-\xi)$ in equations (A.7) and (A.8) to obtain

$$
\begin{aligned}
\left|\Lambda_{1}\right\rangle & =\sqrt{\frac{1}{b}} g_{1}(\xi-1) \frac{1}{(C U-\xi)}|W\rangle \\
\left|\Lambda_{2}\right\rangle & =\sqrt{\frac{1}{b}} g_{2}(\xi-1) \frac{1}{(C U-\xi)}|W\rangle .
\end{aligned}
$$


As we can see the solutions get modified w.r.t. the $B=0$ case, only via possible modifications of the eigenvalue $\xi$. Substitution of these solutions into equations (A.5) and (A.6) gives

$$
\begin{aligned}
& \sqrt{\frac{1}{2 b}}(\xi-1)\left\langle W\left|\frac{1}{C U-\xi}\right| W\right\rangle g_{1}-\frac{A}{\sqrt{2 b}}\left(\xi-1+\frac{b}{A}\right) g_{1}-\frac{2 a A}{\sqrt{6 b}}(\xi-1) g_{2}=0 \\
& \sqrt{\frac{1}{2 b}}(\xi-1)\left\langle W\left|\frac{1}{C U-\xi}\right| W\right\rangle g_{2}-\frac{A}{\sqrt{2 b}}\left(\xi-1+\frac{b}{A}\right) g_{2}+\frac{2 a A}{\sqrt{6 b}}(\xi-1) g_{1}=0
\end{aligned}
$$

The bracket which appears here is the same as the one in [21] and is given by

$$
\left\langle W\left|\frac{1}{C U-\xi}\right| W\right\rangle=V_{00}+\frac{\xi+1}{\xi-1} 2 \Re F(\eta)
$$

where

$$
F(\eta)=\psi\left(\frac{1}{2}+\frac{\eta}{2 \pi i}\right)-\psi\left(\frac{1}{2}\right), \quad \xi=-\frac{1}{1-2 \cosh \eta}[2-\cosh \eta-\mathrm{i} \sqrt{3} \sinh \eta]
$$

$\psi(x)$ is the logarithmic derivative of the Euler $\Gamma$-function.

Substitution of these in (A.11) and (A.12) gives us

$$
\left(\Re F(\eta)-\frac{b}{4}\right) g_{1}-\frac{a A}{\sqrt{3}} \frac{\xi-1}{\xi+1} g_{2}=0, \quad\left(\Re F(\eta)-\frac{b}{4}\right) g_{2}+\frac{a A}{\sqrt{3}} \frac{\xi-1}{\xi+1} g_{1}=0 .
$$

This system of equations will have non trivial solutions for $g_{2}$ and $g_{1}$ if the determinant of the coefficient matrix is zero, i.e.

$$
\frac{b}{4}=\Re F(\eta) \pm a A \tanh \frac{\eta}{2},
$$

Using equations A.15) we can show that $g_{2}= \pm i g_{1}$. This is a constraint on $g_{1}$ and $g_{2}$ thus we cannot split the eigenstates in the two directions, choosing one of the constants to be zero. $g_{1}$ is now an overall constant, which can be chosen real and fixed by normalization completely.

The eigenstates are then

\section{Case-1}

$$
\begin{aligned}
& \frac{b}{4}=\Re F(\eta)+a A \tanh \frac{\eta}{2}, \quad g_{2}=-i g_{1}=-i g_{d}\left(\eta_{1}, \eta_{2}\right) \\
& \left|V^{\left(\xi_{1}\right)}\right\rangle=g_{d}\left(\eta_{1}, \eta_{2}\right)\left(\begin{array}{c}
1 \\
-i \\
\sqrt{\frac{1}{b}}\left(\xi_{1}-1\right) \frac{1}{C U-\xi_{1}}|W\rangle \\
-i \sqrt{\frac{1}{b}}\left(\xi_{1}-1\right) \frac{1}{C U-\xi_{1}}|W\rangle
\end{array}\right)
\end{aligned}
$$




$$
\left|V^{\left(\bar{\xi}_{2}\right)}\right\rangle=g_{d}\left(\eta_{2}, \eta_{1}\right)\left(\begin{array}{c}
1 \\
-i \\
\sqrt{\frac{1}{b}}\left(\bar{\xi}_{2}-1\right) \frac{1}{C U-\bar{\xi}_{2}}|W\rangle \\
-i \sqrt{\frac{1}{b}}\left(\bar{\xi}_{2}-1\right) \frac{1}{C U-\xi_{2}}|W\rangle
\end{array}\right)
$$

\section{Case-2}

$$
\begin{aligned}
& \frac{b}{4}=\Re F(\eta)-a A \tanh \frac{\eta}{2}, \quad g_{2}=i g_{1}=i g_{d}\left(\eta_{2}, \eta_{1}\right) \\
& \left|V^{\left(\xi_{2}\right)}\right\rangle=g_{d}\left(\eta_{2}, \eta_{1}\right)\left(\begin{array}{c}
1 \\
i \\
\sqrt{\frac{1}{b}}\left(\xi_{2}-1\right) \frac{1}{C U-\xi_{2}}|W\rangle \\
i \sqrt{\frac{1}{b}}\left(\xi_{2}-1\right) \frac{1}{C U-\xi_{2}}|W\rangle
\end{array}\right) \\
& \left|V^{\left(\bar{\xi}_{1}\right)}\right\rangle=g_{d}\left(\eta_{1}, \eta_{2}\right)\left(\begin{array}{c}
1 \\
i \\
\sqrt{\frac{1}{b}}\left(\bar{\xi}_{1}-1\right) \frac{1}{C U-\xi_{1}}|W\rangle \\
i \sqrt{\frac{1}{b}}\left(\bar{\xi}_{1}-1\right) \frac{1}{C U-\xi_{1}}|W\rangle
\end{array}\right) \text {. }
\end{aligned}
$$

Normalizing them in the following way ${ }^{9}$

$$
\begin{aligned}
\bar{V}_{\alpha}^{\xi_{i}} V^{\xi_{j}, \alpha} & =\delta^{i j} \\
\bar{V}_{\alpha}^{\bar{\xi}_{i}} V^{\bar{\xi}_{j}, \alpha} & =\delta^{i j} \\
\bar{V}_{\alpha}^{\bar{\xi}} V^{\xi, \alpha} & =0
\end{aligned}
$$

we get, use the results of [21],

$$
\left|g_{d}\left(\eta_{1}, \eta_{2}\right)\right|^{2}=\frac{1}{2 \Delta}\left[\left(1-r\left(\eta_{1}, \eta_{2}\right)\right)+r\left(\eta_{1}, \eta_{2}\right) \sinh \eta_{1} \frac{\partial}{\partial \eta_{1}}\left[\log \Re F\left(\eta_{1}\right)\right]\right)^{-1},
$$

where

$$
r\left(\eta_{1}, \eta_{2}\right)=\Re F\left(\eta_{1}\right) \frac{\tanh \left(\frac{\eta_{1}}{2}\right)+\tanh \left(\frac{\eta_{2}}{2}\right)}{\Re F\left(\eta_{2}\right) \tanh \left(\frac{\eta_{1}}{2}\right)+\Re F\left(\eta_{1}\right) \tanh \left(\frac{\eta_{2}}{2}\right)} .
$$

It is important to notice that $V^{\left(\xi_{1}\right)}$ and $V^{\left(\bar{\xi}_{1}\right)}$ are degenerate eigenstates of $\mathcal{X}$, and the same holds for $V^{\left(\xi_{2}\right)}$ and $V^{\left(\bar{\xi}_{2}\right)}$.

\footnotetext{
${ }^{9}$ This is the standard way to normalize eigenvectors of hermitean matrices
} 


\section{A.2 Continuous spectrum}

If $\xi$ is in the continuous spectrum of $C U(\xi=\nu(k)$, 21]), we cannot invert the operator $(C U-\xi)$. Thus, in this case, the solution of (A.7) and (A.8) is

$$
\begin{gathered}
\left|\Lambda_{1}\right\rangle=A_{1}(k)|k\rangle+\frac{1}{\sqrt{b}} g_{1}(\nu(k)-1) \wp \frac{1}{(C U-\nu(k))}|W\rangle \\
\left|\Lambda_{2}\right\rangle=A_{2}(k)|k\rangle+\frac{1}{\sqrt{b}} g_{2}(\nu(k)-1) \wp \frac{1}{C U-\nu(k)}|W\rangle .
\end{gathered}
$$

Where $\wp$ is the principal value, 21. Using these in (A.5) and (A.6), we get

$$
\begin{aligned}
& A_{1}(k)=g_{1} \sqrt{\frac{2}{b}} k\left(\Re F_{c}(k)-\frac{b}{4}\right)-\frac{\sqrt{2} A a}{\sqrt{3 b}} k\left(\frac{\nu(k)-1}{\nu(k)+1}\right) g_{2} \\
& A_{2}(k)=g_{2} \sqrt{\frac{2}{b}} k\left(\Re F_{c}(k)-\frac{b}{4}\right)+\frac{\sqrt{2} A a}{\sqrt{3 b}} k\left(\frac{\nu(k)-1}{\nu(k)+1}\right) g_{1}
\end{aligned}
$$

Note that in this case $g_{1}$ and $g_{2}$ are completely free and we can choose them to construct two linearly independent orthogonal vectors as follows

Case-1 $\quad g_{2}=i g_{1}=i g_{c}(k)$

$$
V^{1}(k)=g_{c}(k)\left(\begin{array}{c}
1 \\
i \\
P(k)|k\rangle+\frac{1}{\sqrt{b}}(\nu(k)-1) \wp \frac{1}{C U-\nu(k)}|W\rangle-i H(k, a)|k\rangle \\
i P(k)|k\rangle+i \frac{1}{\sqrt{b}}(\nu(k)-1) \wp \frac{1}{C U-\nu(k)}|W\rangle+H(k, a)|k\rangle
\end{array}\right)
$$

Case-2 $g_{2}=-i g_{1}=-i g_{c}(-k)$

$$
V^{2}(k)=g_{c}(-k)\left(\begin{array}{c}
1 \\
P(k)|k\rangle+\frac{1}{\sqrt{b}}(\nu(k)-1) \wp \frac{1}{C U-\nu(k)}|W\rangle+i H(k, a)|k\rangle \\
-i P(k)|k\rangle-i \frac{1}{\sqrt{b}}(\nu(k)-1) \wp \frac{1}{C U-\nu(k)}|W\rangle+H(k, a)|k\rangle
\end{array}\right),
$$

where

$$
P(k)=\sqrt{\frac{2}{b}} k\left(\Re F_{c}(k)-\frac{b}{4}\right), \quad H(k, a)=\frac{\sqrt{2} A a}{\sqrt{3 b}} k\left(\frac{\nu(k)-1}{\nu(k)+1}\right) .
$$

Imposing the continuous orthonormality condition

$$
\bar{V}^{i, \alpha}(k) V_{\alpha}^{j}\left(k^{\prime}\right)=\delta^{i j} \delta\left(k-k^{\prime}\right)
$$

we get

$$
g_{c}(k)=\left[\frac{4 \Delta}{b} N(k)\left(4+k^{2}\left(\Re F_{c}(k)-\frac{b}{4}-\frac{A a}{\tanh \frac{\pi k}{4}}\right)^{2}\right)\right]^{-1 / 2}
$$

Sending $k \rightarrow-k$ we get the right degeneracy for $\mathcal{X}$. 


\section{B Diagonalization of the 3-string vertex and the Lump state}

We can express the oscillators $a_{N, \alpha}^{(r)}$, appearing in the 3-string vertex (3.3), in terms of the oscillators of the diagonal basis as

$$
\begin{gathered}
a_{N, \alpha}^{(r)}=\sum_{i=1}^{2}\left(a_{\xi_{i}}^{(r)} \bar{V}_{N, \alpha}^{\left(\xi_{i}\right)}+a_{\bar{\xi}_{i}}^{(r)} \bar{V}_{N, \alpha}^{\left(\bar{\xi}_{i}\right)}+\int_{-\infty}^{\infty} d k a_{i}^{(r)}(k) \bar{V}_{N, \alpha}^{(i)}(k)\right) \\
a_{N, \alpha}^{(r) \dagger}=\sum_{i=1}^{2}\left(a_{\xi_{i}}^{(r) \dagger} V_{N, \alpha}^{\left(\xi_{i}\right)}+a_{\bar{\xi}_{i}}^{(r) \dagger} V_{N, \alpha}^{\left(\bar{\xi}_{i}\right)}+\int_{-\infty}^{\infty} d k a_{i}^{(r) \dagger}(k) V_{N, \alpha}^{(i)}(k)\right) .
\end{gathered}
$$

Using these oscillators and the fact that $\tau \bar{V}=V\left(\tau_{\beta}^{\alpha}=\left(\begin{array}{cc}1 & 0 \\ 0 & -1\end{array}\right)\right)$, we can rewrite the 3 -string vertex as

$$
\begin{aligned}
\left|V_{3}^{m}\right\rangle=N_{m} & \exp \left[-\frac{1}{2} \sum_{r, s} \sum_{i=1}^{2}\left(a_{\xi_{i}}^{(r) \dagger} \bar{V}_{N, \alpha}^{\left(\xi_{i}\right)}+a_{\bar{\xi}_{i} \dagger}^{(r) \dagger} \bar{V}_{N, \alpha}^{\left(\bar{\xi}_{i}\right)}+\int_{-\infty}^{\infty} d k a_{i}^{(r) \dagger}(k) \bar{V}_{N, \alpha}^{(i)}(k)\right)\left(\tau C^{\prime} \mathcal{X}\right)_{\beta, N M}^{\alpha(r s)}\right. \\
& \left.\times \sum_{j=1}^{2}\left(a_{\xi_{j}}^{(s) \dagger} V_{M}^{\left(\xi_{j}\right) \beta}+a_{\bar{\xi}_{j}}^{(s) \dagger} V_{M}^{\left(\bar{\xi}_{j}\right) \beta}+\int_{-\infty}^{\infty} d k^{\prime} a_{j}^{(s) \dagger}\left(k^{\prime}\right) V_{M}^{(j) \beta}\left(k^{\prime}\right)\right)\right]\left|\Omega_{b, \theta}\right\rangle
\end{aligned}
$$

The twist operator $\tau C^{\prime}$ acts on the eigenstates of the discrete and continous spectra as follows

$$
\tau C^{\prime} V^{\left(\xi_{i}\right)}=V^{\left(\bar{\xi}_{i}\right)} \quad \tau C^{\prime} V^{(i)}(k)=V^{(i+1)}(-k),
$$

where $V^{3}(k)$ is identified with $V^{1}(k)$. Then (B.3) becomes

$$
\begin{gathered}
\left|V_{3}^{m}\right\rangle=N_{m} \exp \left[-\frac{1}{2} \sum_{r, s} \sum_{i=1}^{2}\left(a_{\xi_{i}}^{(r) \dagger} \mu^{r s}\left(\bar{\xi}_{i}\right) a_{\bar{\xi}_{i}}^{(s) \dagger}+a_{\bar{\xi}_{i}}^{(r) \dagger} \mu^{r s}\left(\xi_{i}\right) a_{\xi_{i}}^{(s) \dagger}+\right.\right. \\
\left.\left.+\int_{-\infty}^{\infty} d k a_{i}^{(r) \dagger}(k) \mu^{r s}(-k) a_{i+1}^{(s) \dagger}(-k)\right)\right]\left|\Omega_{b}\right\rangle .
\end{gathered}
$$

In order to write this in an exact diagonal form, we need to introduce oscillators with definite $\tau$-twist parity

$$
\begin{gathered}
e_{\xi_{i}}^{r}=\frac{a_{\xi_{i}}^{r}+a_{\bar{\xi}_{i}}^{r}}{\sqrt{2}}=\frac{a_{\xi_{i}}^{r}+\tau C^{\prime} a_{\xi_{i}}^{r}}{\sqrt{2}}, \quad o_{\xi_{i}}^{r}=-i \frac{a_{\xi_{i}}^{r}-a_{\bar{\xi}_{i}}^{r}}{\sqrt{2}}=-i \frac{a_{\xi_{i}}^{r}-\tau C^{\prime} a_{\xi_{i}}^{r}}{\sqrt{2}} \\
e_{i}^{r}(k)=\frac{a_{i}^{r}(k)+a_{i+1}^{r}(-k)}{\sqrt{2}}=\frac{a_{i}^{r}(k)+\tau C^{\prime} a_{i}^{r}(k)}{\sqrt{2}} \\
o_{i}^{r}(k)=-i \frac{a_{i}^{r}(k)-a_{i+1}^{r}(-k)}{\sqrt{2}}=-i \frac{a_{i}^{r}(k)-\tau C^{\prime} a_{i}^{r}(k)}{\sqrt{2}}
\end{gathered}
$$


These oscillators have the following BPZ conjugation property

$$
\operatorname{bpz} o_{i}=-o_{i}^{\dagger} \quad \operatorname{bpz} e_{i}=-e_{i}^{\dagger},
$$

and satisfy the commutation relations

$$
\begin{gathered}
{\left[e_{\xi_{i}}, e_{\xi_{j}}^{\dagger}\right]=\delta_{i j}, \quad\left[o_{\xi_{i}}, o_{\xi_{j}}^{\dagger}\right]=\delta_{i j},} \\
{\left[e_{i}(k), e_{j}^{\dagger}\left(k^{\prime}\right)\right]=\delta_{i j} \delta\left(k-k^{\prime}\right), \quad\left[o_{i}(k), o_{j}^{\dagger}\left(k^{\prime}\right)\right]=\delta_{i j} \delta\left(k-k^{\prime}\right),}
\end{gathered}
$$

with all the other commutators vanishing. Using them into (B.5) we finally obtain

$$
\begin{gathered}
\left|V_{3}^{m}\right\rangle=N_{m} \exp \left[-\frac{1}{4} \sum_{r, s} \sum_{i=1}^{2}\left(\left[\mu^{r s}\left(\xi_{i}\right)+\mu^{r s}\left(\bar{\xi}_{i}\right)\right]\left(e_{\xi_{i}}^{(r) \dagger} e_{\xi_{i}}^{(s) \dagger}+o_{\xi_{i}}^{(r) \dagger} o_{\xi_{i}}^{(s) \dagger}\right)\right.\right. \\
-i\left[\mu^{r s}\left(\xi_{i}\right)-\mu^{r s}\left(\bar{\xi}_{i}\right)\right]\left(o_{\xi_{i}}^{(r) \dagger} e_{\xi_{i}}^{(s) \dagger}-e_{\xi_{i}}^{(r) \dagger} o_{\xi_{i}}^{(s) \dagger}\right) \\
-\int_{-\infty}^{\infty} d k \mu^{r s}(k)\left(e_{i}^{(r) \dagger}(k) e_{i}^{(s) \dagger}(k)+o_{i}^{(r) \dagger}(k) o_{i}^{(s) \dagger}(k)\right) \\
\left.\left.-i \int_{-\infty}^{\infty} d k \mu^{r s}(k)\left(e_{i}^{(r) \dagger}(k) o_{i}^{(s) \dagger}(k)-o_{i}^{(r) \dagger}(k) e_{i}^{(s) \dagger}(k)\right)\right)\right]\left|\Omega_{b, \theta}\right\rangle
\end{gathered}
$$

This gives the diagonal representation of the 3 -string interaction vertex. The same procedure gives the following diagonal representation of the transverse part of the Lump

$$
\begin{gathered}
\left|S_{\perp}\right\rangle=\frac{A^{2}\left(3+4 a^{2}\right)}{\sqrt{2 \pi b^{3}}(\operatorname{Det} G)^{\frac{1}{4}}} \operatorname{Det}(\mathcal{I}-\mathcal{X})^{\frac{1}{2}} \operatorname{Det}(\mathcal{I}+\mathcal{T})^{\frac{1}{2}} \exp \left(-\frac{1}{2} \sum_{i=1}^{2}\left[t_{d}\left(\eta_{i}\right)\left(e_{\xi_{i}}^{\dagger} e_{\xi_{i}}^{\dagger}+o_{\xi_{i}}^{\dagger} o_{\xi_{i}}^{\dagger}\right)+\right.\right. \\
\left.\left.+\frac{1}{2} \int_{-\infty}^{\infty} d k t_{c}(k)\left(e_{i}^{\dagger}(k) e_{i}^{\dagger}(k)+o_{i}^{\dagger}(k) o_{i}^{\dagger}(k)\right)\right]\right)\left|\Omega_{b, \theta}\right\rangle
\end{gathered}
$$

\section{Asymptotic behaviours}

In Section 3.3, we have analyzed our solution in the large and small limits of the parameter $b$. In this appendix we compute the relevant matrix elements in these asymptotic regimes.

\section{C.1 The $b \rightarrow \infty$ Limit}

From (A.16), we can write

$$
\begin{gathered}
|a|=\frac{\Re F\left(\eta_{2}\right)-\Re F\left(\eta_{1}\right)}{\left[V_{00}+2 \Re F\left(\eta_{2}\right)\right] \tanh \left(\frac{\eta_{1}}{2}\right)+\left[V_{00}+2 \Re F\left(\eta_{1}\right)\right] \tanh \left(\frac{\eta_{2}}{2}\right)} \\
\frac{b}{4}=\frac{\Re F\left(\eta_{2}\right) \tanh \left(\frac{\eta_{1}}{2}\right)+\Re F\left(\eta_{1}\right) \tanh \left(\frac{\eta_{2}}{2}\right)}{\tanh \left(\frac{\eta_{1}}{2}\right)+\tanh \left(\frac{\eta_{2}}{2}\right)}
\end{gathered}
$$


where we take, by definition, $\eta_{2}>\eta_{1}>0$. There are two ways of taking $b \rightarrow \infty$ i) $\eta_{2} \rightarrow \infty$; $\eta_{1}$ fixed

In this limit we can see that

$$
\frac{b}{4} \approx\left(\frac{\tanh \left(\frac{\eta_{1}}{2}\right)}{1+\tanh \left(\frac{\eta_{1}}{2}\right)}\right) \log \left(\eta_{2}\right), \quad a \approx \frac{1}{2 \tanh \left(\frac{\eta_{1}}{2}\right)}>\frac{1}{2} .
$$

ii) $\eta_{2} \rightarrow \infty ; \eta_{1} \rightarrow \infty$

We can parameterize $\eta_{2}=\eta^{y}, \eta_{1}=\eta^{x}$ and then take $\eta \rightarrow \infty$, while keeping $y>x$. We then obtain

$$
\frac{b}{4} \approx \frac{1}{2}(y+x) \log (\eta), \quad a \approx \frac{1}{2} \frac{y-x}{y+x}<\frac{1}{2}
$$

We will be concerned with this second regime as it is the one connected to $a=0$, which is a condition arising from the existence of the critical value for the $E$-field, when $b \rightarrow \infty$. In this second limit it can be easily seen that the discrete eigenvectors have the following behaviour

$$
\begin{gathered}
V_{0}^{\xi_{i}, 1}=V_{0}^{\bar{\xi}_{i}, 1} \approx \frac{1}{\sqrt{2 \Delta}} e^{-\eta_{i} / 2} \sqrt{\eta_{i} \log \eta_{1} \eta_{2}}, \\
V_{0}^{\xi_{i}, 2}=-V_{0}^{\bar{\xi}_{i}, 2} \approx(-1)^{i} \frac{i}{\sqrt{2 \Delta}} e^{-\eta_{i} / 2} \sqrt{\eta_{i} \log \eta_{1} \eta_{2}},
\end{gathered}
$$

and

$$
V_{n}^{\xi_{i}, \alpha} \approx-\frac{V_{0}^{\xi_{i}, \alpha}}{\sqrt{\log \eta_{1} \eta_{2}}}, \quad V_{n}^{\bar{\xi}_{i}, \alpha} \approx-\frac{V_{0}^{\bar{\xi}_{i}, \alpha}}{\sqrt{\log \eta_{1} \eta_{2}}}
$$

For the continuous spectrum the situation is more complicated and getting this limits is not easy. However, it is possible to calculate the limit of $\left(V_{0}^{i, \alpha}(k)\right)^{2}$, which is enough for our purposes. We have

$$
\left(V_{0}^{1,1}(k)\right)^{2}=\left[\frac{4 \Delta}{b} N(k)\left(4+k^{2}\left(\Re F_{c}(k)-\frac{b}{4}-\frac{A a}{\tanh \frac{\pi k}{4}}\right)^{2}\right)\right]^{-1} .
$$

When $b \rightarrow \infty$ this expression vanishes every where except at

$$
k_{0} \approx-\frac{4}{\pi} \operatorname{arctanh}(2 a)
$$

where it diverges. Expanding around $k_{0}$ one easily gets

$$
\left(V_{0}^{1,1}(k)\right)^{2} \approx \Delta^{-1} \frac{4 a}{k_{0}\left(1-4 a^{2}\right) N\left(k_{0}\right)} \frac{\bar{b}}{\pi\left(1+\left(k-k_{0}\right)^{2} \bar{b}^{2}\right.}
$$

where

$$
\bar{b}=\frac{k_{0} \pi\left(1-4 a^{2}\right)}{64 a} b .
$$

Now taking the $b \rightarrow \infty$ limit one obtains

$$
\left(V_{0}^{1,1}(k)\right)^{2} \approx \frac{1}{2 \Delta} \delta\left(k-k_{0}\right) .
$$


Following the same procedure one can also show that

$$
\left(V_{0}^{2,1}(k)\right)^{2} \approx \frac{1}{2 \Delta} \delta\left(k+k_{0}\right)
$$

remember that

$$
\left|V_{0}^{1,2}(k)\right|^{2}=\left(V_{0}^{1,1}(k)\right)^{2}, \quad\left|V_{0}^{2,2}(k)\right|^{2}=\left(V_{0}^{2,1}(k)\right)^{2} .
$$

The non zero components, $V_{m}^{i, \alpha}(k)$, can be expressed in terms of a generating function. For instance, the generating function for $V_{m}^{1,1}(k)$ is given by

$$
F^{(k)}(z)=A_{1}(k) f^{(k)}(z)-\frac{(1-\nu(k)) V_{0}^{1,1}(k)}{\sqrt{b}} B(k, z)
$$

where

$$
\begin{gathered}
A_{1}(k)=V_{0}^{1,1}(k) \sqrt{\frac{2}{b}} k\left(\Re F_{c}(k)-\frac{b}{4}-\frac{A a}{\tanh \left(\frac{\pi k}{4}\right)}\right), \\
B(k, z)=\frac{2}{1-\nu(k)}\left[\Re F_{c}(k)+\frac{\pi}{2 \sqrt{3}} \frac{\nu(k)-1}{\nu(k)+1}+\frac{2 i}{k}+\log (i z)-2 i f(k)(z)\right] \\
+\frac{2}{1-\nu(k)}\left[\Phi\left(e^{-4 i \arctan (z)}, 1,1+\frac{k}{4 i}\right) e^{-4 i \arctan (z)} e^{-k \arctan (z)}\right]
\end{gathered}
$$

where $\Phi$ is the LerchPhi function and $f^{(k)}$ is the generating function for the spectrum of the Neumann matrix without zero modes, 33 . Inverting this equation we can write $V_{m}^{1,1}(k)$ as

$$
V_{m}^{1,1}(k)=A_{1}(k) \frac{\sqrt{m}}{2 \pi i} \oint d z \frac{f^{(k)}(z)}{z^{m+1}}-\frac{(1-\nu(k)) V_{0}^{1,1}(k)}{\sqrt{b}} \frac{\sqrt{m}}{2 \pi i} \oint d z \frac{B(k, z)}{z^{m+1}}
$$

With the same procedure one can also write

$$
V_{m}^{2,1}(k)=A_{1}^{\prime}(k) \frac{\sqrt{m}}{2 \pi i} \oint d z \frac{f^{(k)}(z)}{z^{m+1}}-\frac{(1-\nu(k)) V_{0}^{2,1}(k)}{\sqrt{b}} \frac{\sqrt{m}}{2 \pi i} \oint d z \frac{B(k, z)}{z^{m+1}}
$$

with

$$
A_{1}^{\prime}(k)=V_{0}^{2,1}(k) \sqrt{\frac{2}{b}} k\left(\Re F_{c}(k)-\frac{b}{4}+\frac{A a}{\tanh \left(\frac{\pi k}{4}\right)}\right) .
$$

The other vectors are related to these ones as

$$
V_{n}^{1,2}(k)=i V_{n}^{1,1}(k), \quad V_{n}^{2,2}(k)=-i V_{n}^{2,1}(k)
$$

\section{C.1.1 Limit of $\hat{S}_{m n}^{\alpha \beta(c)}$}

With all these results at hand we can now calculate the continuous spectrum contribution to the non zero mode matrix elements in the limits under consideration. Recalling that spectrum of the Neumann matrix without zero modes is given by

$$
v_{m}^{(k)}=\frac{\sqrt{m}}{2 \pi i} \oint d z \frac{f^{(k)}(z)}{z^{m+1}}
$$


we can write

$$
\hat{S}_{n m}^{11(c)}=\int_{-\infty}^{\infty} d k t_{c}(k)(-1)^{n}\left[V_{n}^{1,1}(k) \bar{V}_{m}^{1,1}(k)+V_{n}^{2,1}(k) \bar{V}_{m}^{2,1}(k)\right]
$$

as

$$
\begin{gathered}
\hat{S}_{m n}^{11(c)}=\int_{-\infty}^{\infty} d k t_{c}(k)(-1)^{m}\left[A_{1}(k) A_{1}(k) v_{m}^{(k)} v_{n}^{(k)}-A_{1}(k) V_{0}^{1,1}(k) v_{m}^{(k)}(1-\bar{\nu}(k)) \tilde{B}_{n}(k) \frac{1}{\sqrt{b}}\right. \\
\left.-A_{1}(k) V_{0}^{1,1}(k) v_{n}^{(k)}(1-\nu(k)) \tilde{B}_{m}(k) \frac{1}{\sqrt{b}}+\left(V_{0}^{1,1}(k)\right)^{2}(1-\bar{\nu}(k))(1-\nu(k)) \tilde{B}_{m}(k) \tilde{B}_{n}(k) \frac{1}{b}\right] \\
+\left[A_{1}(k) \rightarrow A_{1}^{\prime}(k), V_{0}^{1,1}(k) \rightarrow V_{0}^{2,1}(k)\right]
\end{gathered}
$$

where

$$
\tilde{B}_{m}(k)=\frac{\sqrt{m}}{2 \pi i} \oint d z \frac{B(k, z)}{z^{m+1}} .
$$

Note that if the indices are separated by comma then the first index is the label of the vector and the second is the space time index, otherwise both are space time indices. Now we want to calculate each term in the above expression in the limit when $b \rightarrow \infty$. To this end we notice the following

$$
\begin{gathered}
\lim _{b \rightarrow \infty} A_{1}(k) A_{1}(k)=\lim _{b \rightarrow \infty}\left(V_{0}^{1,1}(k)\right)^{2}\left(\frac{2 k^{2}}{b}\right)\left(\Re F_{c}(k)-\frac{b}{4}-\frac{A a}{\tanh \left(\frac{\pi k}{4}\right)}\right)^{2} \\
=\lim _{x \rightarrow-\infty}\left(\frac{k^{2}}{2 \Delta N(k)}\right) \frac{x^{2}}{4+k^{2} x^{2}}=\left(\frac{k^{2}}{2 \Delta N(k)}\right) \frac{1}{k^{2}}=\frac{1}{2 \Delta N(k)}
\end{gathered}
$$

where $x=\left(\Re F_{c}(k)-\frac{b}{4}-\frac{A a}{\tanh \left(\frac{\pi k}{4}\right)}\right)$. The other terms are zero in the limit because, either they contain term like $\left(k-k_{0}\right) \delta\left(k-k_{0}\right)$ in the integral or they are of order $\frac{1}{b}$. Therefore, we are left with

$$
\lim _{b \rightarrow \infty} \hat{S}_{m n}^{11(c)}=\lim _{b \rightarrow \infty} \hat{S}_{m n}^{22(c)}=\Delta^{-1} S_{m n}, \quad \text { where } \quad S_{n m}=-\int_{-\infty}^{\infty} \frac{d k t_{c}(k)}{N(k)} v_{n}^{(k)} v_{m}^{(-k)}
$$

and

$$
\lim _{b \rightarrow \infty} \hat{S}_{m n}^{21(c)}=\lim _{b \rightarrow \infty} \hat{S}_{m n}^{12(c)}=0,
$$

which is the sliver in each direction with corrections of order $\frac{1}{b}$.

\section{C.1.2 Limit of $\hat{S}_{0 m}^{\alpha \beta(c)}$}

In this section we would like to justify that the contribution from the continuous spectrum to $\hat{S}_{0 m}^{\alpha \beta}$ is zero in the limit. This can be computed the same way as before since we have

$$
\lim _{b \rightarrow \infty} \hat{S}_{0 m}^{\alpha \beta(c)}=\lim _{b \rightarrow \infty} \sum_{i=1}^{2} \int_{-\infty}^{\infty} d k t_{c}(k) V_{0}^{i, \alpha}(k) V_{m}^{i, \beta}(k) .
$$


For instance, lets calculate $\hat{S}_{0 m}^{11(c)}$ which is given by

$$
\begin{gathered}
\hat{S}_{0 m}^{11(c)}=\lim _{b \rightarrow \infty} \int_{-\infty}^{\infty} d k t_{c}(k) v_{m}^{(k)} \sqrt{\frac{2}{b}} k\left(\left(V_{0}^{1,1}(k)\right)^{2}\left[\Re F_{c}(k)-\frac{b}{4}-\frac{A a}{\tanh \left(\frac{\pi k}{4}\right)}\right]\right. \\
\left.+\left(V_{0}^{2,1}(k)\right)^{2}\left[\Re F_{c}(k)-\frac{b}{4}+\frac{A a}{\tanh \left(\frac{\pi k}{4}\right)}\right]\right)+O\left(\frac{1}{\sqrt{b}}\right)
\end{gathered}
$$

We have already verified that $\lim _{b \rightarrow \infty}\left(V_{0}^{i, \alpha}(k)\right)^{2} \approx \frac{1}{2} \delta\left(k \pm k_{0}\right)$. This will allow us to expand the terms in square brackets about the points $\pm k_{0}$ to get

$$
\begin{aligned}
\hat{S}_{0 m}^{11(c)}= & \frac{1}{\Delta} \lim _{b \rightarrow \infty} \int_{-\infty}^{\infty} d k t_{c}(k) v_{m}^{(k)}\left[\frac{1}{2} \delta\left(k-k_{0}\right) \sqrt{b}\left(k-k_{0}\right) k_{0} \pi\left(\frac{1-4 a^{2}}{32 a}\right)\right. \\
& \left.+\frac{1}{2} \delta\left(k+k_{0}\right) \sqrt{b}\left(k+k_{0}\right)\left(-k_{0}\right) \pi\left(\frac{1-4 a^{2}}{32 a}\right)\right]+O\left(\frac{1}{\sqrt{b}}\right) .
\end{aligned}
$$

Due to the presence of the delta functions the terms $\left(k \pm k_{0}\right) \sqrt{b}$ are both finite in the $b \rightarrow \infty$ limit. As a matter of this fact we can safely do the integrals first and take the limits later. Since the integrals vanishes we note that

$$
\hat{S}_{0 m}^{11(c)} \approx 0
$$

Similar steps show that all the remaining terms of $\hat{S}_{0 m}^{\alpha \beta(c)}$ are also zero.

\section{C.2 The $b \rightarrow 0$ Limit}

As it was mentioned before this limit can be obtained by taking $\eta_{1} \rightarrow 0$. In this limit it is not hard to see that

$$
\begin{gathered}
b \approx 2 \frac{\Re F\left(\eta_{2}\right)}{\tanh \left(\frac{\eta_{2}}{2}\right)} \eta_{1} \\
g_{d}\left(\eta_{1}, \eta_{2}\right) \approx \frac{1}{\sqrt{2 \Delta}}\left(1-\frac{\tanh \left(\frac{\eta_{2}}{2}\right)}{2 \Re F\left(\eta_{2}\right)} \eta_{1}\right) \\
g_{d}\left(\eta_{2}, \eta_{2}\right) \approx \frac{1}{\sqrt{2 \Delta}}\left[2 \tanh \left(\frac{\eta_{2}}{2}\right)\left(\sinh \eta_{2} \frac{\partial}{\partial \eta_{2}}\left[\log \Re F\left(\eta_{2}\right)\right]-1\right)\right]^{-1 / 2} \sqrt{\eta_{1}}
\end{gathered}
$$

One can use these results and equations (A.18) through (A.22) to write down $V_{0}^{\xi_{i}, \alpha}, V_{0}^{\bar{\xi}_{i}, \alpha}$ $V_{n}^{\xi_{i}, \alpha}$ and $V_{n}^{\bar{\xi}_{i}, \alpha}$ as

$$
\begin{gathered}
V_{0}^{\xi_{1}, 1}=V_{0}^{\bar{\xi}_{1}, 1} \approx \frac{1}{\sqrt{2 \Delta}}\left(1-\frac{\tanh \left(\frac{\eta_{2}}{2}\right)}{2 \Re F\left(\eta_{2}\right)} \eta_{1}\right), \\
V_{0}^{\xi_{1}, 2}=-V_{0}^{\bar{\xi}_{1}, 2} \approx-i \frac{1}{\sqrt{2 \Delta}}\left(1-\frac{\tanh \left(\frac{\eta_{2}}{2}\right)}{2 \Re F\left(\eta_{2}\right)} \eta_{1}\right), \\
V_{0}^{\xi_{2}, 1}=V_{0}^{\bar{\xi}_{2}, 1} \approx \frac{1}{\sqrt{2 \Delta}}\left[2 \tanh \left(\frac{\eta_{2}}{2}\right)\left(\sinh \eta_{2} \frac{\partial}{\partial \eta_{2}}\left[\log \Re F\left(\eta_{2}\right)\right]-1\right)\right]^{-1 / 2} \sqrt{\eta_{1}}
\end{gathered}
$$




$$
V_{0}^{\xi_{2}, 2}=-V_{0}^{\bar{\xi}_{2}, 2} \approx i \frac{1}{\sqrt{2 \Delta}}\left[2 \tanh \left(\frac{\eta_{2}}{2}\right)\left(\sinh \eta_{2} \frac{\partial}{\partial \eta_{2}}\left[\log \Re F\left(\eta_{2}\right)\right]-1\right)\right]^{-1 / 2} \sqrt{\eta_{1}},
$$

and

$$
\begin{gathered}
V_{n}^{\xi_{1}, \alpha}= \pm V_{n}^{\bar{\xi}_{1}, \alpha} \approx \sqrt{\eta_{1}} \\
V_{n}^{\xi_{2}, \alpha}= \pm V_{n}^{\bar{\xi}_{2}, \alpha} \approx \frac{1}{\sqrt{2 \Delta}}\left[2 \tanh \left(\frac{\eta_{2}}{2}\right)\left(\sinh \eta_{2} \frac{\partial}{\partial \eta_{2}}\left[\log \Re F\left(\eta_{2}\right)\right]-1\right)\right]^{-1 / 2} f\left(\eta_{2}\right) .
\end{gathered}
$$

The $f$ is a regular function of $\eta_{2}$. On the other hand

$$
g_{c}(k) \approx 0 .
$$

This shows all $V_{0}^{i, \alpha}(k)$ are zero, whereas $V_{m}^{i, \alpha}(k)$ are finite and $b$ independent to the leading order. These results are extensively used in section 3.3 to calculate quantities like $s_{1}, s_{2}$ in the $b \rightarrow 0$ limit.

\section{References}

[1] E. Witten, "Noncommutative Geometry And String Field Theory," Nucl. Phys. B 268 (1986) 253.

[2] Kazuki Ohmori, A Review on Tachyon Condensation in open string theory, arXiv:hep-th/0102085.

[3] I. Y. Arefeva, D. M. Belov, A. A. Giryavets, A. S. Koshelev and P. B. Medvedev, "Noncommutative field theories and (super)string field theories," arXiv:hep-th/0111208.

[4] Washington Taylor and Barton Zwieback, D-Branes, Tachyons, and String Field Theory arXiv:hep-th/0311017.

[5] A. Sen, "Tachyon dynamics in open string theory," arXiv:hep-th/0410103.

[6] J. Kluson, "Time dependent solution in open bosonic string field theory," arXiv:hep-th/0208028. M. Fujita and H. Hata, "Time dependent solution in cubic string field theory," JHEP 0305 (2003) 043 arXiv:hep-th/0304163. I.Ya.Aref'eva, L.V.Joukovskaya, A.S.Koshelev, Time Evolution in Superstring Field Theory on nonBPS brane.I. Rolling Tachyon and Energy-Momentum Conservation, JHEP 0309 (2003) 012 arXiv:hep-th/0301137.

[7] Theodore G. Erler, David J. Gross Locality, Causality, and an Initial Value Formulation for Open String Field Theory, arXiv:hep-th/0406199.

[8] T. G. Erler, "Level truncation and rolling the tachyon in the lightcone basis for open string field theory," arXiv:hep-th/0409179. 
[9] L. Rastelli, A. Sen and B. Zwiebach, "Vacuum string field theory," arXiv:hep-th/0106010

[10] D.Gaiotto, L.Rastelli, A.Sen and B.Zwiebach, Ghost Structure and Closed Strings in Vacuum String Field Theory, [hep-th/0111129].

[11] G. Moore and W. Taylor The singular geometry of the sliver, JHEP 0201 (2002) 004 [hep-th/0111069].

[12] Y. Okawa, "Some exact computations on the twisted butterfly state in string field theory,” JHEP 0401 (2004) 066 arXiv:hep-th/0310264.

[13] L. Bonora, C. Maccaferri and P. Prester, "Dressed sliver solutions in vacuum string field theory," JHEP 0401 (2004) 038 arXiv:hep-th/0311198.]

[14] L. Bonora, C. Maccaferri and P. Prester, "The perturbative spectrum of the dressed sliver," Phys. Rev. D 71 (2005) 026003 arXiv:hep-th/0404154.

[15] Masako Fujita, Hiroyuki Hata, Rolling Tachyon Solution in Vacuum String Field Theory, Phys. Rev. D 70 (2004) 086010 arXiv:hep-th/0403031.

[16] L. Bonora, C. Maccaferri, R. J. Scherer Santos and D. D. Tolla, "Exact time-localized solutions in vacuum string field theory," arXiv:hep-th/0409063

[17] P. Mukhopadhyay and A. Sen, "Decay of unstable D-branes with electric field," JHEP 0211, 047 (2002) arXiv:hep-th/0208142.

[18] L. Bonora, C. Maccaferri, R. Scherer Santos, D.D. Tolla "Fundamental strings in SFT," arXiv:hep-th/0501111.

[19] N. Seiberg, L. Susskind and N. Toumbas, "Strings in background electric field, space/time noncommutativity and a new noncritical string theory," JHEP 0006 (2000) 021 arXiv:hep-th/0005040.

[20] B. Feng, Y. H. He and N. Moeller, Zeeman Spectroscopy of the Star Algebra, JHEP 0205 (2002) 041 [arXiv: hep-th/0203175. B. Chen and F. L. Lin, "Star spectroscopy in the constant B field background," Nucl. Phys. B 637 (2002) 199 arXiv:hep-th/0203204.

[21] D.M.Belov, Diagonal Representation of Open String Star and Moyal Product, [hepth/0204164].

[22] F.Sugino, Witten's Open String Field Theory in Constant B-field Background, JHEP 0003, (2000) 017 [hepth9912254]

[23] T. Kawano and T. Takahashi, "Open string field theory on noncommutative space," Prog. Theor. Phys. 104 (2000) 459 arXiv:hep-th/9912274.

[24] E. Fuchs, M. Kroyter and A. Marcus, "Squeezed State Projectors in String Field Theory." JHEP 0211 (2002) 046 arXiv:hep-th/0207001, M. Ihl, A. Kling and S. Uhlmann, String field theory projectors for fermions of integral weight, JHEP 0403 
(2004) 002 arXiv:hep-th/0312314, S. Uhlmann, "A note on kappa-diagonal surface states," JHEP 0411 (2004) 003 arXiv:hep-th/0408245, E. Fuchs and M. Kroyter, "On surface states and star-subalgebras in string field theory," JHEP 0410 (2004) 004 arXiv:hep-th/0409020.

[25] A. Sen, "Rolling tachyon," JHEP 0204 (2002) 048 arXiv:hep-th/0203211. A. Sen, "Tachyon matter," JHEP 0207 (2002) 065 arXiv:hep-th/0203265 . A. Sen, "Time evolution in open string theory," JHEP 0210 (2002) 003 arXiv:hep-th/0207105. A. Sen, "Time and tachyon," Int. J. Mod. Phys. A 18 (2003) 4869 arXiv:hep-th/0209122. A. Sen, "Open and closed strings from unstable D-branes," arXiv:hep-th/0305011. A. Sen, "Open-closed duality: Lessons from matrix model," arXiv:hep-th/0308068. A. Sen Rolling Tachyon Boundary State, Conserved Charges and Two Dimensional String Theory, JHEP 0405 (2004) 076 [arXiv:hep-th//0402157].

[26] I. R. Klebanov, J. Maldacena and N. Seiberg, "D-brane decay in two-dimensional string theory,” JHEP 0307 (2003) 045 arXiv:hep-th/0305159.

[27] N. Lambert, H. Liu and J. Maldacena, "Closed strings from decaying D-branes," arXiv:hep-th/0303139.

[28] D. Gaiotto, N. Itzhaki and L. Rastelli, "Closed strings as imaginary D-branes," arXiv:hep-th/0304192.

[29] V.A.Kostelecky and R.Potting, Analytical construction of a nonperturbative vacuum for the open bosonic string, Phys. Rev. D 63 (2001) 046007 [hep-th/0008252].

[30] L.Rastelli, A.Sen and B.Zwiebach, Classical solutions in string field theory around the tachyon vacuum, Adv. Theor. Math. Phys. 5 (2002) 393 [hep-th/0102112].

[31] L. Bonora, D. Mamone and M. Salizzoni, B field and squeezed states in Vacuum String Field Theory, Nucl. Phys. B630 (2002) 163 [hep-th/0201060]. L. Bonora, D. Mamone and M. Salizzoni, Vacuum String Field Theory with B field, JHEP 0204 (2002) 020 [hep-th/0203188]. L. Bonora, D. Mamone and M. Salizzoni, Vacuum String Field Theory ancestors of the GMS solitons, JHEP 0301 (2003) 013 [hep-th/0207044].

[32] Ashoke Sen, Energy Momentum Tensor and Marginal Deformations in Open String Field Theory, arXiv:hep-th/0403200.

[33] L.Rastelli, A.Sen and B.Zwiebach, Star Algebra Spectroscopy, JHEP 0203 (2002) 029 [hep-th/0111281].

[34] D.J.Gross and A.Jevicki, Operator Formulation of Interacting String Field Theory, Nucl.Phys. B283 (1987) 1.

[35] N. Ohta, "Covariant Interacting String Field Theory In The Fock Space Representation," Phys. Rev. D 34 (1986) 3785 [Erratum-ibid. D 35 (1987) 2627].

[36] L. Bonora, C. Maccaferri, D. Mamone and M. Salizzoni, "Topics in string field theory," arXiv:hep-th/0304270. 
[37] A.Leclair, M.E.Peskin, C.R.Preitschopf, String Field Theory on the Conformal Plane. (I) Kinematical Principles, Nucl.Phys. B317 (1989) 411. 